\title{
The Lick AGN Monitoring Project 2011: Dynamical Modeling of the Broad-line Region
}

\author{
Peter R. Williams ${ }^{1}$ (1) , Anna Pancoast ${ }^{2,21}$ (1) , Tommaso Treu $^{1}$ (1), Brendon J. Brewer ${ }^{3}$, Aaron J. Barth ${ }^{4}$ (1), Vardha N. Bennert $^{5}$ (1),
}

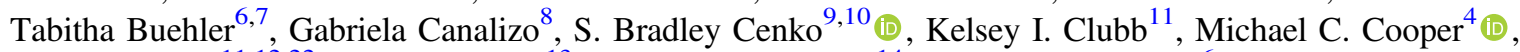
Alexei V. Filippenko ${ }^{11,12,22}$ (1) , Elinor Gates $^{13}$, Sebastian F. Hoenig ${ }^{14}{ }^{10}$, Michael D. Joner ${ }^{6}$, Michael T. Kandrashoff ${ }^{11}$, Clifton David Laney ${ }^{15}$ (1), Mariana S. Lazarova ${ }^{16}$, Weidong $\mathrm{Li}^{11,23}$, Matthew A. Malkan ${ }^{1}$ (1), Jacob Rex ${ }^{11}$, Jeffrey M. Silverman ${ }^{11,17}$ (1), Erik Tollerud ${ }^{18}$ (1) , Jonelle L. Walsh ${ }^{19}$ (D), and Jong-Hak Woo $^{20}$ (1)

${ }^{1}$ Department of Physics and Astronomy, University of California, Los Angeles, CA 90095-1547, USA; pwilliams@astro.ucla.edu

${ }^{2}$ Harvard-Smithsonian Center for Astrophysics, 60 Garden Street, Cambridge, MA 02138, USA

${ }^{3}$ Department of Statistics, The University of Auckland, Private Bag 92019, Auckland 1142, New Zealand

${ }^{4}$ Department of Physics and Astronomy, 4129 Frederick Reines Hall, University of California, Irvine, CA 92697-4575, USA

${ }^{5}$ Physics Department, California Polytechnic State University San Luis Obispo, CA 93407, USA

${ }^{6}$ Department of Physics and Astronomy, N283 ESC, Brigham Young University, Provo, UT 84602-4360, USA

${ }_{8}^{7}$ Department of Physics and Astronomy, University of Utah, Salt Lake City, UT 84112, USA

${ }^{8}$ Department of Physics and Astronomy, University of California, Riverside, CA 92521, USA

9 Astrophysics Science Division, NASA Goddard Space Flight Center, Mail Code 661, Greenbelt, MD 20771, USA

${ }^{10}$ Joint Space-Science Institute, University of Maryland, College Park, MD 20742, USA

${ }^{11}$ Department of Astronomy, University of California, Berkeley, CA 94720-3411, USA

${ }^{12}$ Miller Institute for Basic Research in Science, University of California, Berkeley, CA 94720, USA

${ }^{13}$ Lick Observatory, P.O. Box 85, Mount Hamilton, CA 95140, USA

${ }^{14}$ Department of Physics \& Astronomy, University of Southampton, Southampton, SO17 1BJ, UK

${ }^{15}$ Department of Physics and Astronomy, Western Kentucky University, 1906 College Heights Boulevard, Bowling Green, KY 42101, USA ${ }^{16}$ University of Northern Colorado, Greeley, CO 80639, USA

${ }^{17}$ Samba TV, San Francisco, CA 94107, USA

${ }^{18}$ Space Telescope Science Institute, USA

${ }^{19}$ George P. and Cynthia Woods Mitchell Institute for Fundamental Physics and Astronomy, Department of Physics and Astronomy, Texas A\&M University, College Station, TX 77843-4242, USA

${ }^{20}$ Physics and Astronomy Department, Seoul National University, Seoul, 08826, Republic of Korea

Received 2018 June 13; revised 2018 August 30; accepted 2018 September 6; published 2018 October 15

\begin{abstract}
We present models of the $\mathrm{H} \beta$-emitting broad-line region (BLR) in seven Seyfert 1 galaxies from the Lick AGN Monitoring Project 2011 sample, drawing inferences on the BLR structure and dynamics as well as the mass of the central supermassive black hole. We find that the BLR is generally a thick disk, viewed close to face-on, with preferential emission back toward the ionizing source. The dynamics in our sample range from near-circular elliptical orbits to inflowing or outflowing trajectories. We measure black hole masses of $\log _{10}\left(M_{\mathrm{BH}} / M_{\odot}\right)=6.48_{-0.18}^{+0.21}$ for PG $1310-108,7.50_{-0.18}^{+0.25}$ for Mrk 50, 7.46-0.21 for Mrk 141, 7.58 $8_{-0.08}^{+0.08}$ for Mrk 279, 7.11 $1_{-0.17}^{+0.20}$ for Mrk 1511, 6.65 $5_{-0.15}^{+0.27}$ for NGC 4593, and $6.94_{-0.14}^{+0.14}$ for Zw 229-015. We use these black hole mass measurements along with crosscorrelation time lags and line widths to recover the scale factor $f$ used in traditional reverberation mapping measurements. Combining our results with other studies that use this modeling technique, which brings our sample size to 16 , we calculate a scale factor that can be used for measuring black hole masses in other reverberation mapping campaigns. When using the root-mean-square (rms) spectrum and using the line dispersion to measure the line width, we find $\log _{10}\left(f_{\text {rms, } \sigma}\right)_{\text {pred }}=0.57 \pm 0.19$. Finally, we search for correlations between $f$ and other AGN and BLR parameters and find marginal evidence that $f$ is correlated with $M_{\mathrm{BH}}$ and the BLR inclination angle, but no significant evidence of a correlation with the AGN luminosity or Eddington ratio.
\end{abstract}

Key words: galaxies: active - galaxies: nuclei - galaxies: Seyfert

\section{Introduction}

Supermassive black holes are thought to play an important role in galaxy formation and evolution. Tight correlations in the local universe between black hole masses and host-galaxy properties (e.g., Magorrian et al. 1998; Ferrarese \& Merritt 2000; Gebhardt et al. 2000) suggest a fundamental link between the growth of black holes and their hosts. Depending on the relative timing of black hole and host-galaxy growth, one might expect an evolution of these scaling relations, so accurate measurements of black hole masses across cosmic

\footnotetext{
21 Einstein Fellow.

22 Miller Senior Fellow.

${ }^{23}$ Deceased 2011 December 12.
}

time are essential for testing the predictions of different evolutionary scenarios.

In nearby galaxies, black hole masses can be measured through stellar or gas kinematics within the black hole sphere of influence (e.g., Kormendy \& Richstone 1995; Ferrarese \& Ford 2005), but this is not possible at distances greater than $\sim 100 \mathrm{Mpc}$ where even the largest black holes' spheres of influence cannot be resolved. The technique of reverberation mapping (Blandford \& McKee 1982; Peterson 1993) substitutes time resolution for spatial resolution by measuring the response of broad emission lines to active galactic nucleus (AGN) continuum variations, enabling measurements out to cosmological distances. The time lag $\tau$ between continuum and emission-line variations can be combined with the speed of light to obtain a characteristic radius of the broad-line region 
(BLR), while the line width measures the velocity $v$ of the emitting gas. By assuming that the motion of the gas in the BLR is dominated by the black hole's gravity, one can make a virial estimate of the black hole's mass,

$$
M_{\mathrm{BH}}=f \frac{c \tau v^{2}}{G},
$$

where $f$ is a scale factor of order unity that accounts for the detailed structure, orientation, and dynamics of the BLR. Typically, an average value of $f$ is used, found by aligning AGNs with the $M_{\mathrm{BH}}-\sigma_{*}$ relation for quiescent galaxies (e.g., Onken et al. 2004; Collin et al. 2006; Woo et al. 2010, 2013; Graham et al. 2011; Grier et al. 2013a; Batiste et al. 2017). The scatter in the $M_{\mathrm{BH}}-\sigma_{*}$ relation introduces an uncertainty of $\sim 0.4$ dex for individual $M_{\mathrm{BH}}$ measurements (Park et al. 2012), making it the largest source of uncertainty in reverberation mapping $M_{\mathrm{BH}}$ measurements. It is therefore very important to understand reverberation mapping results and the $f$ factor; they calibrate all of the single-epoch black hole masses throughout the universe (e.g., Shen \& Kelly 2010).

Since there are multiple ways to measure the line width, more than one version of $f$ exists. Typically, either the line dispersion ( $\sigma_{\text {line }}$, second central moment of the emission-line profile) is measured using the root-mean-square (rms) spectrum, or the FWHM is measured in the time-averaged spectrum. In cases where the rms spectrum is unavailable, such as in single-epoch measurements, the line dispersion measured in the time-averaged spectrum is often used. For clarity, we will specify which $f$ we are discussing by using the notation $f_{s, v}$, where $s$ is the spectrum used (mean or rms) and $v$ is the type of line width (FWHM or $\sigma_{\text {line }}$ ).

The cross-correlation and single-epoch techniques yield a BLR size, but they do not provide information about the gas structure or dynamics needed to determine $f$ for an individual AGN. Recently, high-quality reverberation mapping data sets have enabled velocity-resolved analyses that look individually at how different parts of the broad emission line change, allowing inferences to be drawn about the structure and dynamics of the BLR (Bentz et al. 2009b; Denney et al. 2009a, 2010; Barth et al. 2011a, 2011b; Grier et al. 2013b; Du et al. 2016; Pei et al. 2017). These results are generally consistent with inflowing gas or elliptical orbits, but some have shown signs of gas outflow (Denney et al. 2009b; Du et al. 2016). Other studies have used the code MEMECHO (Horne et al. 1991; Horne 1994) to recover the two-dimensional (2D) transfer function, which defines how continuum changes map to broad-line flux variations as a function of line-of-sight velocity and time delay (Bentz et al. 2010; Grier et al. 2013b; Skielboe et al. 2015). On their own, the resulting maps do not provide details of the BLR structure and kinematics, but can be compared to the transfer functions that result from specific BLR models.

Recent efforts have aimed to measure $M_{\mathrm{BH}}$ independent of $f$ by modeling the structure and dynamics of the BLR directly (Brewer et al. 2011b; Pancoast et al. 2011, 2012, 2014a; Li et al. 2013). Pancoast et al. (2014b, hereafter P14) used the model of Pancoast et al. (2014a) to model the BLR of five AGNs in the Lick AGN Monitoring Project 2008 (LAMP 2008) sample (Bentz et al. 2009b; Walsh et al. 2009), and Grier et al. (2017, hereafter G17) expanded the sample by modeling four AGNs from a 2010 campaign carried out at MDM Observatory. The results from these analyses find an $\mathrm{H} \beta$ - emitting BLR that is a thick disk with kinematics that are best described by a combination of elliptical orbits and inflowing gas, consistent with the velocity-resolved reverberation mapping methods. They also measure scale factors for all of the AGNs in their samples and find a mean scale factor of $\log _{10}\left(\bar{f}_{\mathrm{rms}, \sigma}\right)=0.54 \pm 0.17$ (G17), which is consistent with previous measurements made using the $M_{\mathrm{BH}}-\sigma_{*}$ relation.

In this paper, we expand the sample of AGNs modeled using the techniques of Pancoast et al. (2014a) from 9 to 16 by analyzing the data for seven of the AGNs from the Lick AGN Monitoring Project 2011 campaign (LAMP 2011; Barth et al. 2015). This nearly doubles the sample of AGNs analyzed using this method and will help uncover general trends in BLR properties. We also aim to gain a better understanding of how $f$ is related to other AGN and BLR properties. Since $f$ measurements so far are mostly based on local low-luminosity Seyfert galaxies, understanding how $f$ depends on (for example) continuum luminosity and Eddington ratio will help reduce uncertainties when reverberation mapping techniques are extrapolated and applied to AGNs across the entire universe. In Section 2, we describe the spectroscopic and photometric monitoring data and discuss the spectral decomposition method of extracting $\mathrm{H} \beta$ from the rest of the AGN spectrum. Section 3 summarizes the geometrical and dynamical model from Pancoast et al. (2014a) that we used to model the BLR. We discuss in Section 4 the modeling results for each individual AGN in our sample. In Section 5, we combine our results with those of $\mathrm{P} 14$ and G17 to calculate a mean scale factor $\bar{f}$ and look for useful correlations between $f$ and other parameters. Finally, we conclude in Section 6.

\section{Data}

The data used in this paper were taken as part of LAMP 2011 (Barth et al. 2015). Photometric monitoring of the AGNs was carried out in the Johnson $V$-band using several telescopes: the $0.76 \mathrm{~m}$ Katzman Automatic Imaging Telescope (KAIT) at Lick Observatory (Filippenko et al. 2001); the $0.91 \mathrm{~m}$ telescope at West Mountain Observatory; the $2 \mathrm{~m}$ Faulkes Telescope North at Mt. Haleakala Hawaii and the Faulkes Telescope South at Siding Spring Australia, both part of the Las Cumbres Observatory network (LCO; Brown et al. 2013); the $0.60 \mathrm{~m}$ Super-LOTIS telescope at the Steward Observatory, Kitt Peak; and the Palomar $1.5 \mathrm{~m}$ telescope at Palomar Observatory (Cenko et al. 2006).

Spectra were obtained over the course of 69 nights from 2011 March 27 to June 13 with the Kast double spectrograph mounted on the Shane $3 \mathrm{~m}$ telescope at Lick Observatory. Owing to poor weather, a substantial fraction of the nights were lost. This analysis only uses the spectra from the blue side of the Kast spectrograph, which covered $3440-5515 \AA$ at $1.02 \AA$ per pixel. The spectra were calibrated between nights using the procedure of van Groningen \& Wanders (1992), assuming the flux of the [O III] doublet remained constant throughout the campaign. In addition to the LAMP 2011 observations, Mrk 50 received 12 additional observations from 2011 January through March, and Zw 229-015 received three additional observations after the campaign in order to extend the light curve. All additional observations were also taken with the Kast double spectrograph.

In total, 15 AGNs were observed during the campaign, but only seven had sufficient data quality, and continuum and $\mathrm{H} \beta$ variations for the analysis in this paper. The general properties 
Table 1

AGN and Data Properties

\begin{tabular}{|c|c|c|c|c|c|c|c|c|c|}
\hline Galaxy & Alt. Name & $z$ & $N_{\text {spec }}$ & $N_{\text {phot }}$ & $\begin{array}{c}(\Delta t)_{\mathrm{spec}} \\
\text { (days) }\end{array}$ & $\begin{array}{c}(\Delta t)_{\mathrm{phot}} \\
\text { (days) }\end{array}$ & $\begin{array}{c}(\mathrm{S} / \mathrm{N}) / \mathrm{pix} \\
\mathrm{K} 10\end{array}$ & $\begin{array}{c}(\mathrm{S} / \mathrm{N}) / \mathrm{pix} \\
\text { BG92 }\end{array}$ & $\begin{array}{c}(\mathrm{S} / \mathrm{N}) / \mathrm{pix} \\
\mathrm{VC0} 4\end{array}$ \\
\hline Mrk 50 & & 0.0234 & 55 & 170 & 2.6 & 0.9 & 19.1 & 19.2 & 19.8 \\
\hline Mrk 141 & & 0.0417 & 36 & 93 & 1.7 & 1.1 & 14.5 & 14.1 & 14.6 \\
\hline Mrk 279 & PG $1351+695$ & 0.0305 & 34 & 64 & 2.3 & 1.7 & 17.9 & 18.3 & 18.2 \\
\hline Mrk 1511 & NGC 5940 & 0.0339 & 40 & 71 & 1.9 & 1.5 & 22.6 & 21.7 & 22.4 \\
\hline NGC 4593 & Mrk 1330 & 0.0090 & 43 & 75 & 1.8 & 1.1 & 27.9 & 28.0 & 29.2 \\
\hline Zw 229-015 & & 0.0279 & 29 & 69 & 3.2 & 2.4 & 5.7 & 5.7 & 5.3 \\
\hline PG $1310-108$ & II SZ 10 & 0.0343 & 35 & 63 & 2.2 & 1.6 & 21.4 & 21.5 & 20.3 \\
\hline
\end{tabular}

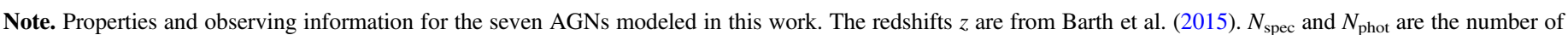

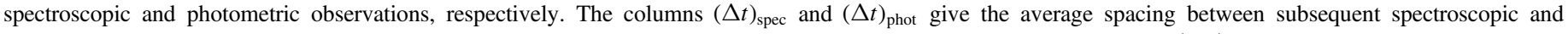

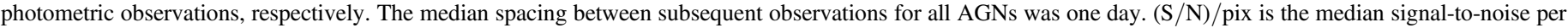
pixel in the $\mathrm{H} \beta$ spectrum from spectral decomposition using the three Fe II templates discussed in Section 2.1 .

of the targets analyzed in this paper along with information on their observations are given in Table 1.

\subsection{Spectral Decomposition}

When we fit BLR models to the data, we allow not only for variations in the total $\mathrm{H} \beta$ flux, but also variations in the detailed shape of the $\mathrm{H} \beta$ broad emission-line spectrum. Because of this, it is critical to disentangle the $\mathrm{H} \beta$ emission from other features contributing to the AGN spectrum. In particular, features such as $\mathrm{He}$ I or Fe II emission that preferentially affect the red wing of the $\mathrm{H} \beta$ profile must be properly subtracted, otherwise the models will attempt to fit an asymmetry that is not intrinsic to the broad $\mathrm{H} \beta$ line. In order to accurately isolate the $\mathrm{H} \beta$ profile, we fit for contributions in the vicinity of $\mathrm{H} \beta$ that may strongly affect its shape-AGN continuum; host-galaxy starlight; [O III] $\lambda 4959$ and $\lambda 5007$; He II $\lambda 4686 ; \mathrm{HeI} \lambda 4471, \lambda 4922$, and $\lambda 5016$; and Fe II emission blends. These components were fit to the spectra by minimizing $\chi^{2}$ with the Levenberg-Marquardt routines in the IDL package MPFIT (Markwardt 2009). After subtracting these features from the data, we are left with the residual $\mathrm{H} \beta$ spectrum. The full details of how the spectra were decomposed into their individual components are given by Barth et al. (2015).

The full fitting procedure was carried out three times for each spectrum using three different $\mathrm{Fe}$ II templates from Boroson \& Green (1992), Kovačević et al. (2010), and Véron-Cetty et al. (2004), hereafter BG92, K10, and VC04, respectively. In addition to free parameters for the velocity shift and broadening kernel, the BG92 and VC04 templates each have one free parameter describing the flux normalization. The K10 template has five components and so has five normalization parameters, making it more flexible, in general. For each AGN, we show distributions of the reduced $\chi^{2}$ values from fitting each epoch in Figure 1. The reduced $\chi^{2}$ values do not include any systematic uncertainties from (for example) flux calibration, but they allow for a relative comparison between the templates in order to determine which one provides the best fit to the data. Generally, the distributions of the reduced $\chi^{2}$ are similar for each template, which we take to mean that each decomposition is equally valid. We choose to run the dynamical modeling procedure using the spectra from all three decompositions and combine the resulting model parameter posterior samples, weighting each run equally. An exception to this is Mrk 50, for which the VC04 template produced poor fits for some epochs. For this object, we adopt only the decompositions that use the K10 and BG92 templates.
Table 2

Model Parameters and Priors

\begin{tabular}{ll}
\hline \hline Parameter & Prior \\
\hline$\mu$ & $\operatorname{LogUniform}\left(1.02 \times 10^{-3}\right.$ lt-days, $\left.\Delta t_{\text {data }}\right)$ \\
$\beta$ & Uniform $(0,2)$ \\
$F$ & Uniform $(0,1)$ \\
$\theta_{i}$ & Uniform $\left(\cos \theta_{i}(0, \pi / 2)\right)$ \\
$\theta_{o}$ & Uniform $(0, \pi / 2)$ \\
$\kappa$ & Uniform $(-0.5,0.5)$ \\
$\gamma$ & Uniform $(1,5)$ \\
$\xi$ & Uniform $(0,1)$ \\
$M_{\text {BH }}$ & $\operatorname{LogUniform~}\left(2.78 \times 10^{4}, 1.67 \times 10^{9} M_{\odot}\right)$ \\
$f_{\text {ellip }}$ & Uniform $(0,1)$ \\
$f_{\text {flow }}$ & Uniform $(0,1)$ \\
$\sigma_{\rho, \text { circ }}$ & $\operatorname{LogUniform}(0.001,0.1)$ \\
$\sigma_{\Theta, \text { circ }}$ & $\operatorname{LogUniform}(0.001,0.1)$ \\
$\sigma_{\rho, \text { radial }}$ & $\operatorname{LogUniform}(0.001,0.1)$ \\
$\sigma_{\Theta, \text { radial }}$ & $\operatorname{LogUniform}(0.001,0.1)$ \\
$\sigma_{\text {turb }}$ & $\operatorname{LogUniform}(0.001,0.1)$ \\
$\theta_{e}$ & Uniform $(0, \pi / 2)$ \\
\hline
\end{tabular}

Note. List of BLR model parameters and their corresponding priors.

In Figure 2, we show the results of the spectral decomposition for each AGN. The left panels show the individual components for the mean spectrum, found using the K10 Fe II template. We note that there is a degeneracy between the He I $\lambda 4922$ and $\lambda 5016 \AA$ lines and two features in the Fe II templates, which appears in the fit for $\mathrm{Zw} 229-015$. However, as discussed by Barth et al. (2015), the sum of these two features is well determined, so the residual $\mathrm{H} \beta$ profile is robust. On the right, we show the mean $\mathrm{H} \beta$ profile derived with the $\mathrm{K} 10 \mathrm{Fe}$ II template as well as the difference in $\mathrm{H} \beta$ profiles from using the other Fe II templates. The thick black bar shows the mean uncertainty in the spectra across the modeled wavelength range. The difference between templates exceeds the flux uncertainty for some AGNs, indicating that template choice is an important factor that may influence the modeling results. In particular, Mrk 1511 and NGC 4593 both have prominent Fe II emission and therefore the $\mathrm{Fe}$ II fits inherently have a strong effect on the resulting $\mathrm{H} \beta$ profile. For objects such as Mrk 141, Mrk 1511, and NGC 4593, the K10 template fits give a mean $\mathrm{H} \beta$ profile with a stronger red wing and weaker blue wing than the BG92 and VC04 template fits. Asymmetries in the line profile are caused by asymmetries in the BLR properties, so we 

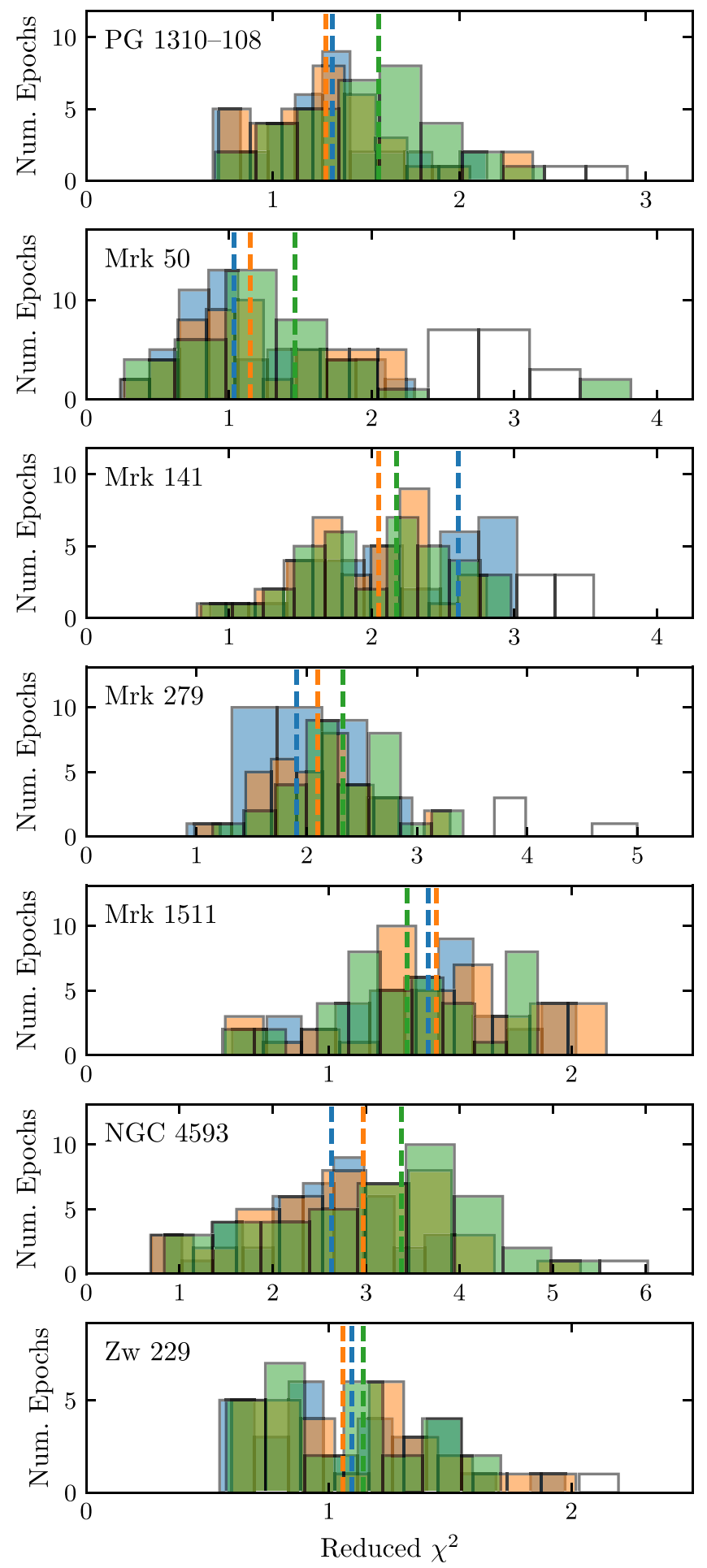

Figure 1. Distribution of the reduced $\chi^{2}$ values for fits to the spectra using the K10 (blue), BG92 (orange), and VC04 (green) Fe II templates. The vertical dashed lines indicate the median reduced $\chi^{2}$ value.

might expect discrepancies in the inferred model asymmetry parameters for these objects.

\section{The Geometric and Dynamical Model of the Broad-line Region}

We use a simply parameterized phenomenological model of the BLR, described by Pancoast et al. (2014a), to model the
AGN data sets. In this framework, the $\mathrm{H} \beta$-emitting BLR is modeled as a distribution of point particles around a central ionizing continuum source that we take to be point-like in nature and isotropically emitting. Each particle receives the continuum emission after a time lag determined by its position, and then is assumed to instantaneously reprocess the light and re-emit it in the direction of the observer. The wavelength of the re-emitted light is centered on $\mathrm{H} \beta$, with a Doppler shift determined by the particle's velocity. By feeding a continuum light curve through the model, we can produce a time series of spectra that we can then directly compare to data.

In order to calculate the line emission of a given BLR geometry at arbitrary times, we need to know the AGN continuum flux at arbitrary times before the time of emission. To determine the continuum flux between data points, we model the AGN continuum using Gaussian processes, which has been shown to be a sufficiently good model for AGN light curves (Kelly et al. 2009; Kozłowski et al. 2010; MacLeod et al. 2010; Zu et al. 2011, 2013; Kozłowski 2016). As discussed by Skielboe et al. (2015), with the use of more general descriptions of the driving light curves, our inferences are robust with respect to the assumption of Gaussian processes, since Gaussian processes are effectively used as a flexible interpolator. This has the advantage of allowing us to include the uncertainties of the continuum modeling in the uncertainties of our model parameters. Additionally, we can extrapolate the continuum light curve to times before and after continuum monitoring in order to model the BLR response for the full extent of the spectroscopic monitoring campaign.

The full details of the BLR model and its limitations are discussed by Pancoast et al. (2014a), but we summarize the main components below. The priors for all model parameters are listed in Table 2.

\subsection{Geometry}

We first assign radial positions to each particle drawn from a Gamma distribution which has a probability density function (PDF)

$$
p(x \mid \alpha, \theta) \propto x^{\alpha-1} \exp \left(-\frac{x}{\theta}\right),
$$

where $\alpha$ is the shape parameter and $\theta$ is the scale parameter. We set a minimum radius of the BLR by shifting the distribution from the origin by the Schwarzschild radius $R_{s}=2 G M_{\mathrm{BH}} / c^{2}$ plus a free parameter $r_{\text {min. }}$. We also set a maximum BLR radius $r_{\text {out }}=c \Delta t_{\text {data }} / 2$, where $\Delta t_{\text {data }}$ is the time between the first modeled point of the AGN continuum light curve and the first spectrum of the broad emission line. This comes from the assumption that our observational campaign is sufficiently long to measure the response of the whole BLR. We then perform a change of variables from $\left(\alpha, \theta, r_{\min }\right)$ to $(\mu, \beta, F)$ :

$$
\begin{gathered}
\mu=r_{\min }+\alpha \theta, \\
\beta=\frac{1}{\sqrt{\alpha}}, \\
F=\frac{r_{\text {min }}}{r_{\text {min }}+\alpha \theta},
\end{gathered}
$$

where $\mu$ is the mean radius, $\beta$ determines the shape of the Gamma distribution, and $F$ is the minimum radius in units of $\mu$. In this framework, the standard deviation of the radial 

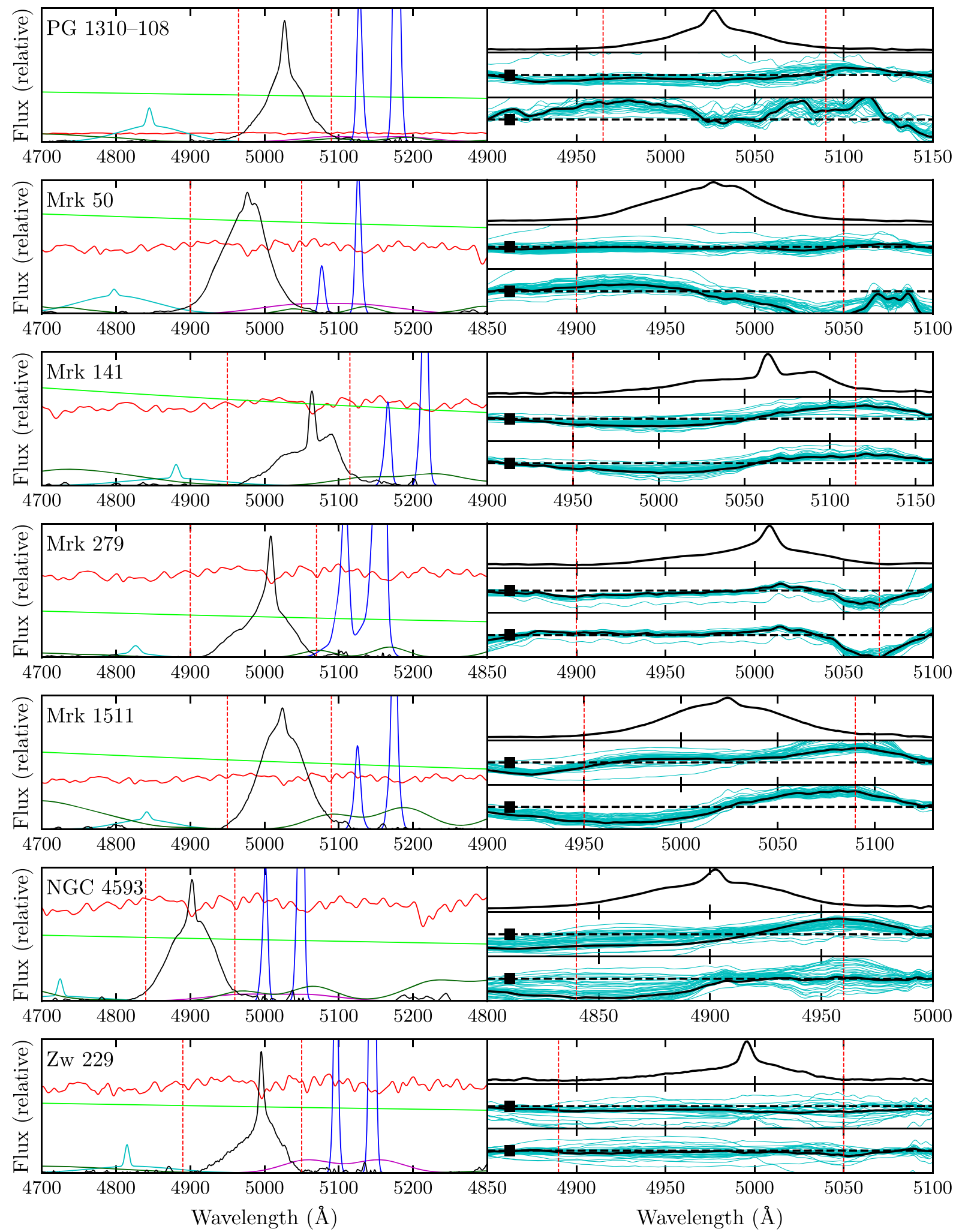

Figure 2. Left panel: spectral decomposition using the K10 Fe II template. The components shown are starlight in red, the AGN featureless continuum in lime green, $\mathrm{He}$ I in magenta, He II in cyan, [O III] in blue, Fe II in dark green, and the residual $\mathrm{H} \beta$ in black. The vertical dashed red lines indicate the wavelength range that was used when fitting the BLR model. Right-top panel: the mean $\mathrm{H} \beta$ profile shape, for reference in the right-middle and right-bottom panels. Right-middle panel: in black is the mean $\mathrm{H} \beta$ spectrum found using the $\mathrm{K} 10 \mathrm{Fe}$ II template minus the mean $\mathrm{H} \beta$ spectrum found using the BG92 template. The cyan lines show the same thing, but for every observational epoch. The black bar on the left shows the mean uncertainty in the spectra over the modeled wavelength range. Right-bottom panel: same as the right-middle panel, but for the $\mathrm{K} 10$ and VC04 templates. For the full spectral decompositions, including the full model fits and residual spectra, see Barth et al. (2015). 
distribution is given by $\sigma_{r}=(1-F) \mu \beta$. The distribution of particles is then "puffed up" out of a plane by an opening angle $\theta_{o}$ such that $\theta_{o}=0^{\circ}$ corresponds to a flat disk and $\theta_{o}=90^{\circ}$ corresponds to a sphere, and the plane of the distribution is inclined by an angle $\theta_{i}$ relative to the observer where $\theta_{i}=0^{\circ}$ is face-on and $\theta_{i}=90^{\circ}$ is edge-on.

The relative emission from each particle is weighted by a parameter

$$
W(\phi)=\frac{1}{2}+\kappa \cos (\phi),
$$

which allows for BLR asymmetry. The angle $\phi$ is measured from the particle to the origin to the observer, and $\kappa$ is a free parameter between -0.5 and 0.5 . In this setup, $\kappa \rightarrow 0.5$ corresponds to emission from the near side of the BLR and $\kappa \rightarrow-0.5$ corresponds to emission from the far side. These cases can physically be interpreted as gas that preferentially reemits away from or back toward the ionizing source, respectively. The broad-line emission is allowed to preferentially come from the faces of the disk according to a parameter $\gamma$, which has a uniform prior between 1 and 5. The angle between a point particle and the disk is

$$
\theta=\operatorname{acos}\left(\cos \theta_{o}+\left(1-\cos \theta_{o}\right) U^{\gamma}\right),
$$

where $U$ is drawn randomly from a uniform distribution between 0 and 1 . When $\gamma \rightarrow 1$, point particles are evenly distributed, and when $\gamma \rightarrow 5$, particles are clustered near the faces of the disk. Finally, the accretion disk is allowed to be transparent to opaque according to the parameter $\xi$, ranging from 0 to 1 . When $\xi \rightarrow 0$, the midplane is opaque, and when $\xi \rightarrow 1$, the midplane is transparent.

\subsection{Dynamics}

The particle velocities are assigned based on the mass of the black hole, their radial position, and the parameters $f_{\text {ellip }}, f_{\text {flow }}$, $\theta_{e}$, and $\sigma_{\text {turb}}$. First, each particle is assigned to have its radial and tangential velocities drawn from a distribution centered either around the circular velocity or around the radial escape velocity. The fraction of particles that are assigned near-circular orbits is given by $f_{\text {ellip }}$, which has a uniform prior between 0 and 1. The specific radial and tangential velocities of these particles are drawn from a Gaussian distribution centered on the circular velocity in the $v_{r}-v_{\phi}$ plane, with standard deviations $\sigma_{\rho, \text { circ }}$ and $\sigma_{\Theta, \text { circ }}$.

The remaining particles are then assigned to be either inflowing or outflowing according to a binary parameter $f_{\text {flow, }}$, where $0<f_{\text {flow }}<0.5$ corresponds to inflow and $0.5<f_{\text {flow }}<1$ corresponds to outflow. The specific radial and tangential velocities for the inflowing and outflowing particles are drawn from Gaussian distributions centered on the inflowing and outflowing escape velocities (respectively) in the $v_{r}-v_{\phi}$ plane, with standard deviations $\sigma_{\rho \text {,radial }}$ and $\sigma_{\Theta \text {,radial }}$. The angle $\theta_{e}$ then rotates the centers of these two distributions toward the circular orbit by an angle between $0^{\circ}$ and $90^{\circ}$ to allow for further flexibility. This means that as $\theta_{e} \rightarrow 90^{\circ}$, all particles approach a distribution centered on the circular velocity, regardless of the value of $f_{\text {ellip. }}$.
Finally, the $\sigma_{\text {turb }}$ parameter allows for random macroturbulent velocities according to

$$
v_{\text {turb }}=\mathcal{N}\left(0, \sigma_{\text {turb }}\right)\left|v_{\text {circ }}\right|,
$$

where $\mathcal{N}\left(0, \sigma_{\text {turb }}\right)$ is a Gaussian distribution with mean 0 and standard deviation $\sigma_{\text {turb }}$, and $v_{\text {turb }}$ is added to the line-of-sight velocity. The parameter $\sigma_{\text {turb }}$ has a log-uniform prior between 0.001 and 0.1 .

\subsection{Producing Emission-line Spectra and Comparing to Data}

Given the continuum light-curve model and a model of the BLR, we can generate $\mathrm{H} \beta$ emission-line spectra at arbitrary times. For each particle, we use the position and line-of-sight velocity to calculate the Doppler and gravitational redshifts and then use the strength of the continuum and the particle's emissivity properties to calculate the amount of flux contribution from that particle. After combining the contributions from all particles, we blur the spectrum by the resolution of the instrument, $\Delta \lambda_{\text {dis }}$, which is calibrated by comparing the width of the observed [O III] $\lambda 5007$ emission line from spectral decomposition, $\Delta \lambda_{\mathrm{obs}}$, to the intrinsic line width, $\Delta \lambda_{\text {true }}$, taken from Whittle (1992):

$$
\Delta \lambda_{\text {dis }}^{2} \approx \Delta \lambda_{\text {obs }}^{2}-\Delta \lambda_{\text {true }}^{2}
$$

Finally, the AGN redshift is left as a free parameter with a Gaussian prior having standard deviation $0.25-0.5 \AA$, depending on the AGN.

We use a Gaussian likelihood function to compare the observed time series of $\mathrm{H} \beta$ spectra from the spectral decomposition to the spectra produced by the model. To explore the parameter space of the continuum light-curve model and BLR model, we use the diffusive nested sampling code DNEST3 (Brewer et al. 2011a). In addition to producing posterior PDFs, diffusive nested sampling also calculates the normalization term, the "evidence," which allows for model comparison.

In practice, our simplified BLR model is unable to reproduce all of the details of the emission line and its fluctuations to within the small spectral uncertainties. We account for this systematic uncertainty by softening the likelihood function with a "temperature" $T$, where $T \geqslant 1$. We divide the $\log$ of the likelihood by $T$, which is equivalent to multiplying the spectral uncertainties by $\sqrt{T}$ in the case of a Gaussian likelihood function. When the temperature is too small, the continuum model hyperparameters are overfitted or the model is unable to efficiently explore parameter space. We choose the lowest temperature for which this is not the case. In our sample, we use $T=150-180$ for Mrk 50, $T=30-40$ for Mrk 141, $T=60-140$ for Mrk 279, $T=20-25$ for Mrk 1511, $T=35$ for PG 1310-108, $T=150-200$ for NGC 4593, and $T=70$ for $\mathrm{Zw} 229-015$. We test the convergence of the model by looking at the samples from the first and second halves of the run and ensuring that they both follow the same distribution.

\section{Results}

In this section, we discuss the dynamical modeling results for our sample of seven LAMP 2011 AGNs. For each AGN, we show a geometric model of the BLR from the posterior sample, chosen to be typical of the geometries in the full posterior sample (Figure 3). We also show randomly chosen model fits to the AGN $\mathrm{H} \beta$ profile, the integrated $\mathrm{H} \beta$ flux light 

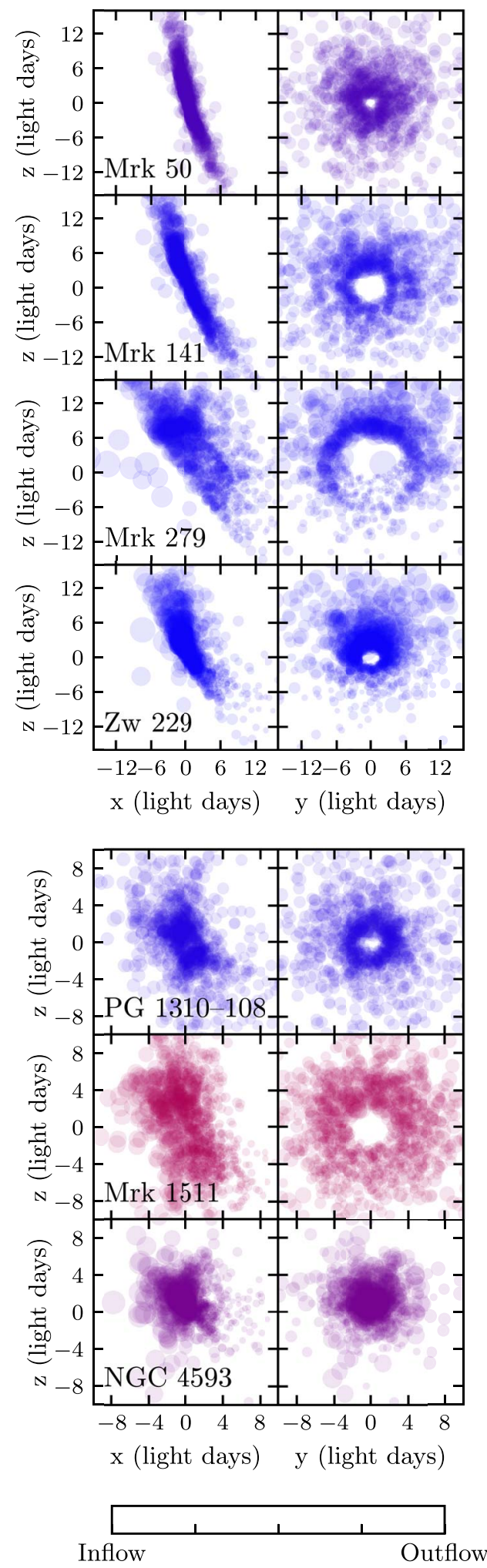

Figure 3. Geometries of the $\mathrm{H} \beta$-emitting BLR for each object, drawn from the posterior samples. In each panel, the observer is viewing the BLR from the positive $x$-axis. Each circle corresponds to one point particle in the model, and the size of the circle corresponds to the amount of line emission coming from that particle. The left panels show an edge-on view, while the right panels show a face-on view. The geometries are color-coded to indicate whether the BLR dynamics exhibit inflow or outflow.

curve, and the continuum light curve in Figure 4. In Figure 5, we show velocity-resolved transfer functions for each AGN, created using the same model as in Figure 3.
In Figures 6-12, we give the posterior distributions of the key model parameters. We also include a parameter to summarize whether the overall dynamics indicate inflowing or outflowing gas, defined such that 1 and -1 are purely radial outflow and inflow, respectively:

$$
\text { In. }- \text { Out. }=\operatorname{sgn}\left(f_{\text {flow }}-0.5\right) \times\left(1-f_{\text {ellip }}\right) \times \cos \left(\theta_{e}\right),
$$

where sgn is the sign function. The median values and $68 \%$ confidence intervals for all parameters are summarized in Table 3. When the posterior PDFs are one-sided, we give upper and lower $68 \%$ confidence limits.

\section{1. $P G 1310-108$}

The top two panels of Figure 4 show how the $\mathrm{H} \beta$ line profile changed over the course of the observing campaign for both the data and one of the models drawn randomly from the posterior sample. The model fits the data very well in the core of the line, but misses some of the detailed structure in the blue wing. This is also visible in the third panel, where we show a model fit to the $\mathrm{H} \beta$ spectrum for one epoch. In the fourth panel, we show the integrated $\mathrm{H} \beta$ flux over the course of the campaign. The $\mathrm{H} \beta$ light curve for PG 1310-108 is relatively short, owing to the loss of many nights to poor weather, but there is a clear variability signal. The models were able to fit the overall shape of the $\mathrm{H} \beta$ light curve, but the details are poorly modeled.

The posterior distributions in Figure 6 give a radial distribution of the PG $1310-108 \mathrm{H} \beta$ emission that is steeper than exponential with a shape parameter $\beta=1.23_{-0.21}^{+0.19}$ for the Gamma distribution. The distribution is shifted from the origin by a minimum radius $r_{\min }=0.96_{-0.40}^{+0.54}$ lt-days and has a mean radius $r_{\text {mean }}=4.5_{-1.0}^{+1.4} \mathrm{lt}$-days and a radial width $\sigma_{r}=4.4_{-1.1}^{+1.5}$ lt-days. The mean lag is $\tau_{\text {mean }}=4.6_{-1.0}^{+1.4}$ days, which is smaller than the cross-correlation measurement $\tau_{\text {cen }}=7.20_{-3.11}^{+2.41}$ days from A. J. Barth et al. (2018, in preparation), but is consistent to within the uncertainties. We note that the uncertainties on both $\tau_{\text {mean }}$ and $\tau_{\text {cen }}$ are relatively high. It is possible that the true $\mathrm{H} \beta$ lag is longer than measured, and that the short spectroscopic monitoring campaign biases our results toward shorter lags. The distributions for the opening and inclination angles exhibit multiple solutions. The $\mathrm{H} \beta$-emitting region is inferred to be either a thick disk with opening angle near $50^{\circ}$ or spherical with opening angle approaching $90^{\circ}$. An example of the former is shown in Figure 3.

There is no preference for emission to be concentrated near the faces of the disk $\left(\gamma=3.0_{-1.3}^{+1.3}\right)$. Despite the median and $68 \%$ confidence interval suggesting only a slight preference for emission from the far side of the BLR $\left(\kappa=-0.17_{-0.17}^{+0.10}\right)$, almost none of the posterior samples have $\kappa>0$, ruling out the possibility of preferential emission from the near side. Solutions with a transparent BLR midplane are preferred slightly over those with an opaque midplane $\left(\xi=0.70_{-0.33}^{+0.23}\right)$.

Dynamically, PG $1310-108$ is best described by models in which few particles are in near-circular orbits $\left(f_{\text {ellip }}<0.28\right)$. The remaining particles are on outflowing orbits, given by $f_{\text {flow }}=0.75_{-0.18}^{+0.16}$, where $f_{\text {flow }}$ is a binary parameter with $f_{\text {flow }}<0.5$ indicating inflow and $f_{\text {flow }}>0.5$ indicating outflow. The full posterior PDF shows almost no solutions with inflow. The outflowing orbits have velocities drawn from a distribution whose center is rotated $\theta_{e}=26_{-18}^{+26}$ degrees from the radial escape velocity toward the circular velocity; thus, more than half of the orbits are actually bound. Finally, there is a small 

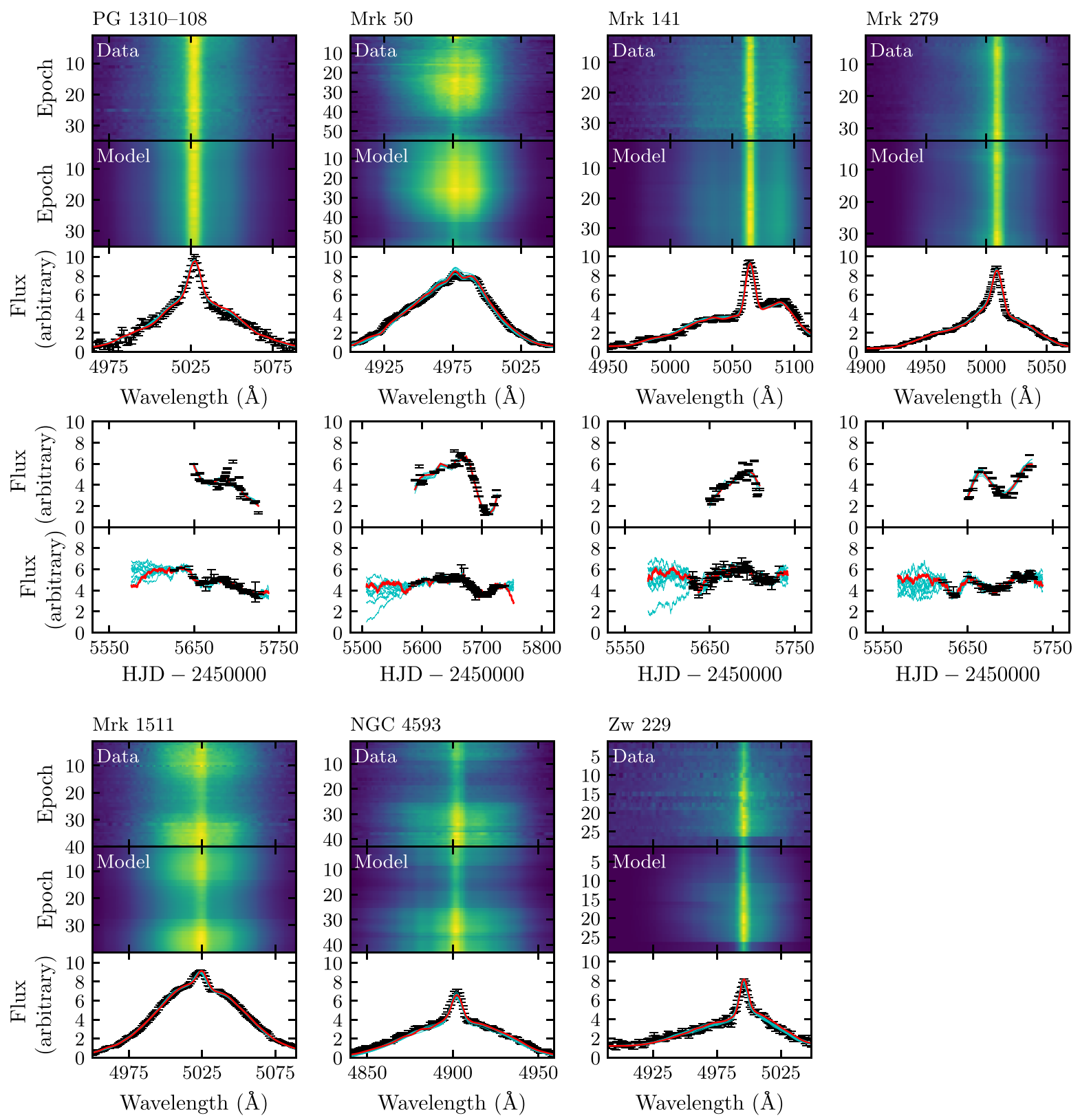

Zw 229
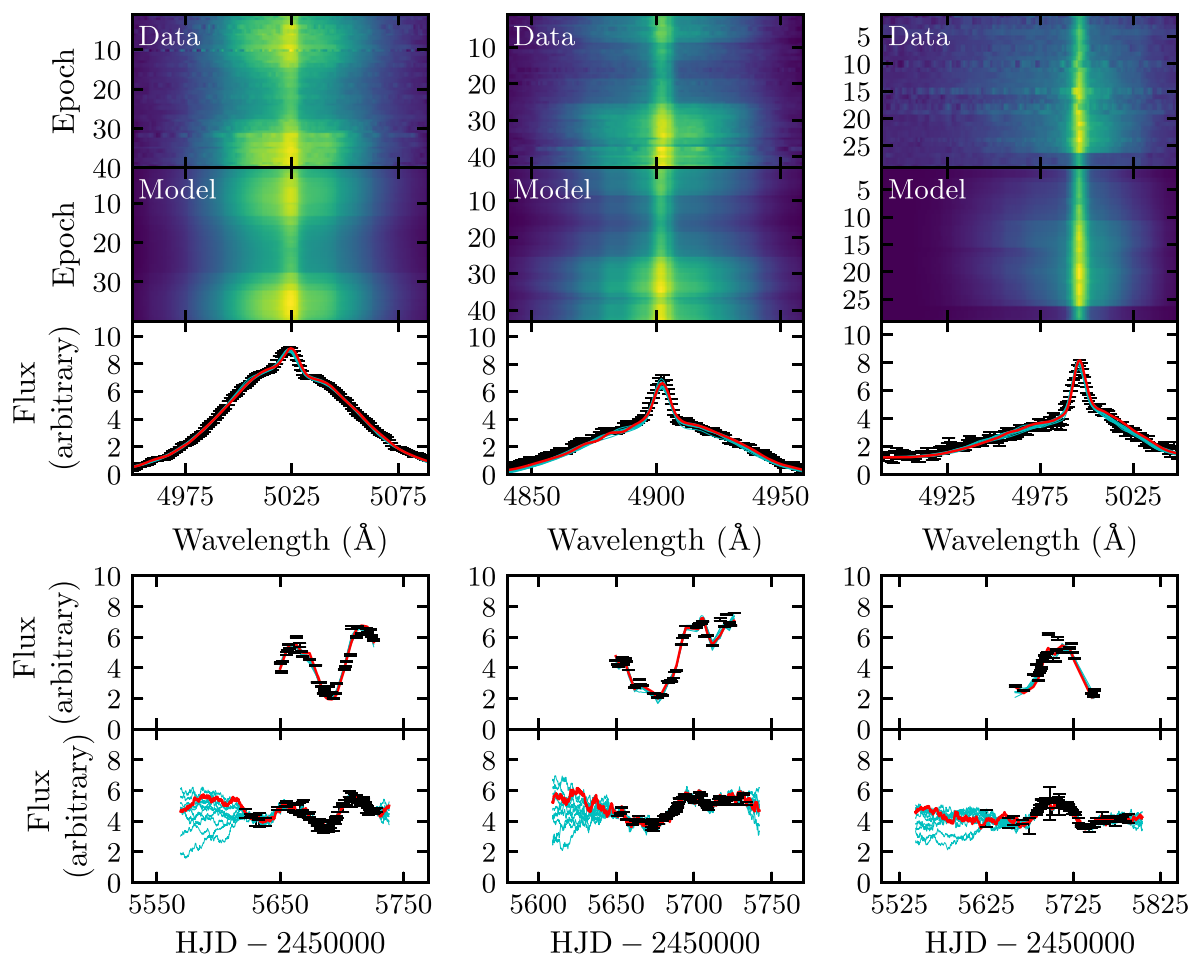

Figure 4. Model fits to the $\mathrm{H} \beta$ line profile, integrated $\mathrm{H} \beta$ flux, and AGN continuum flux. From left to right, the panels show models for PG 1310-108, Mrk 50, Mrk 141, Mrk 279, Mrk 1511, NGC 4593, and Zw 229-015. Within each panel, numbered 1-5 from top to bottom, we have the following. Panels 1 and 2: the observed $\mathrm{H} \beta$ emission-line profile by observation epoch and the profile produced by one sample of the BLR and continuum model. Panel 3: the observed $\mathrm{H} \beta$ profile of one randomly chosen epoch (black), and the corresponding profile (red) produced by the model in Panel 2 . The cyan lines show the $\mathrm{H} \beta$ profile produced by three other randomly chosen models. Panels 4 and 5: time series of the observed integrated $\mathrm{H} \beta$ and continuum flux (black), and the model fits to these light curves (red), corresponding to the model shown in Panel 2. The cyan lines show five other model examples.

contribution from macroturbulent velocities, with $\sigma_{\text {turb }}=$ $0.021_{-0.018}^{+0.049}$ times the circular velocity.

The preference for outflow is visible in the transfer function (Figure 5), in which there is a slight upward-angled structure.
This is a signature one would expect for radially outflowing gas (see, e.g., Welsh \& Horne 1991), indicating that the particles with the shortest lags, which are the particles directly between the ionizing source and the observer, are preferentially 

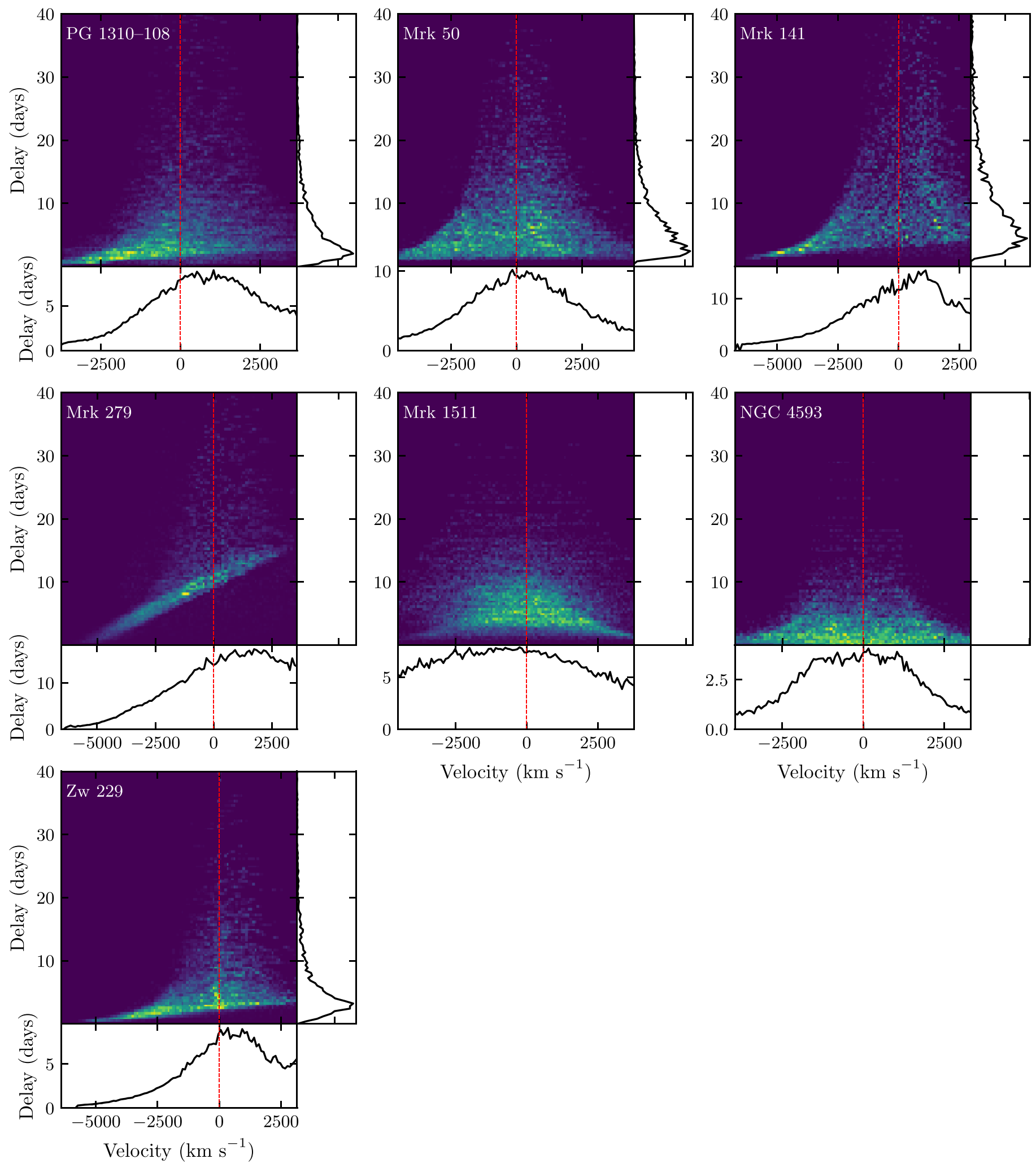

Figure 5. Velocity-resolved transfer functions for each AGN, drawn from the posterior samples and selected to be representative of the full posterior samples. In the right-hand panels, we show the velocity-integrated transfer function, and the bottom panel shows the average time lag for each velocity pixel.

blueshifted, while those with the longest lags on the far side of the source are preferentially redshifted.

The black hole mass for PG $1310-108$ is found to be $\log _{10}\left(M_{\mathrm{BH}} / M_{\odot}\right)=6.48_{-0.18}^{+0.21}$. This value was previously measured by Schulze \& Wisotzki (2010) and is reported in
Busch et al. (2014) as $\log _{10}\left(M_{\mathrm{BH}} / M_{\odot}\right)=7.33 \pm 0.3$. Their measurement was made using the BLR radius estimated from the BLR size-luminosity relationship (Bentz et al. 2009a) combined with the line dispersion and mean scale factor $f_{\text {mean }, \sigma}=3.85 \pm 1.15$ from Collin et al. (2006). Our results 

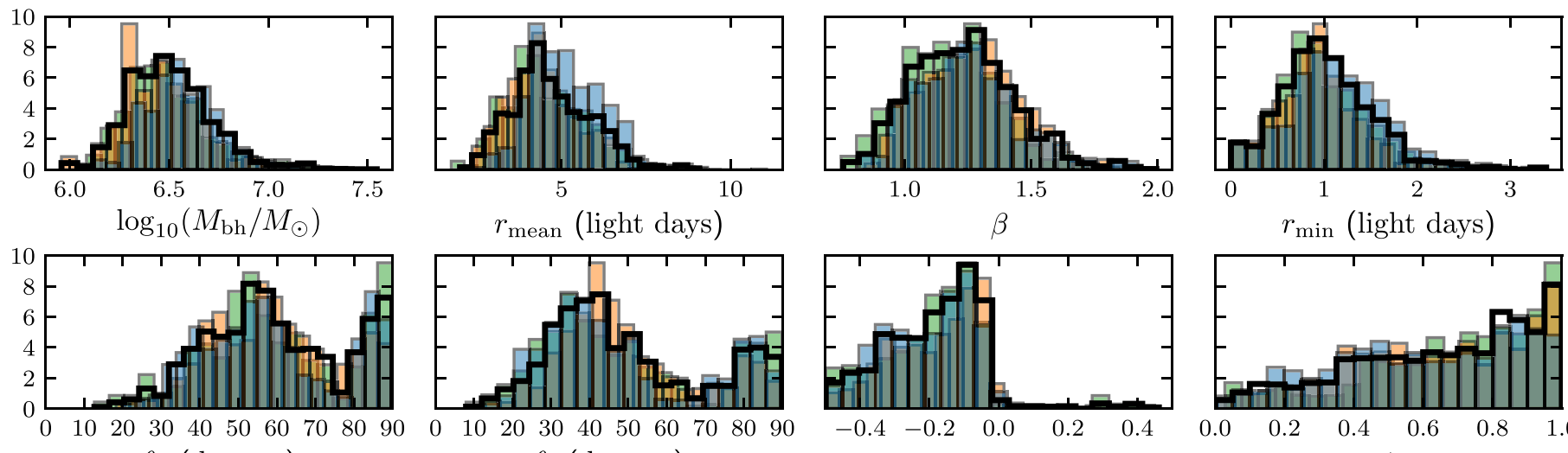

$\beta$
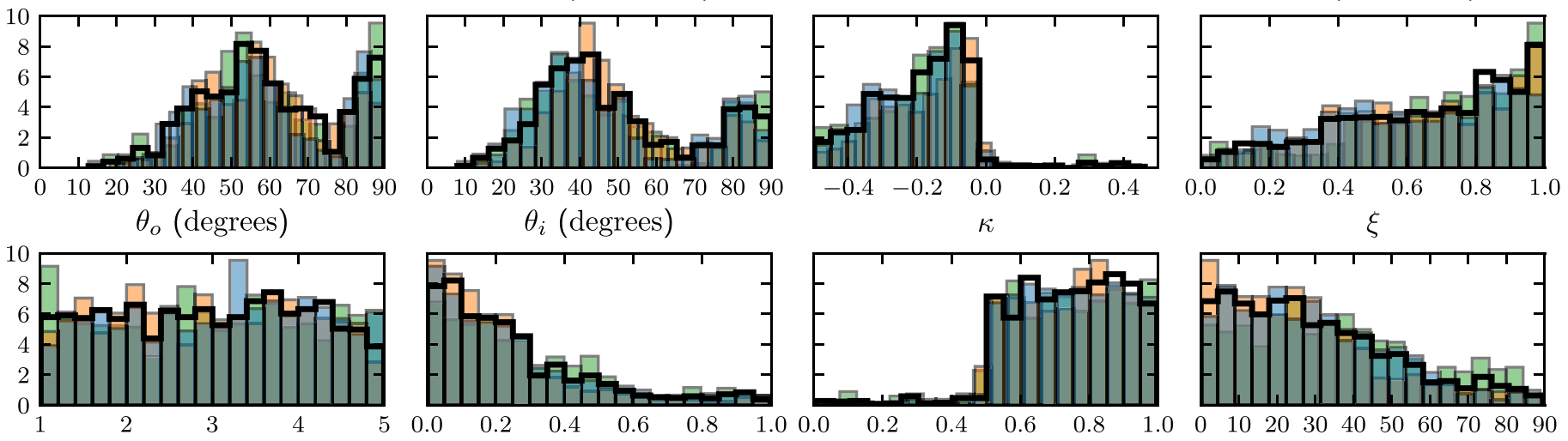

$\gamma$

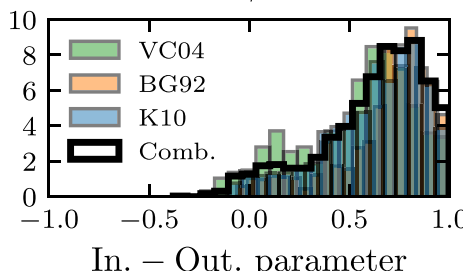

$f_{\text {ellip }}$
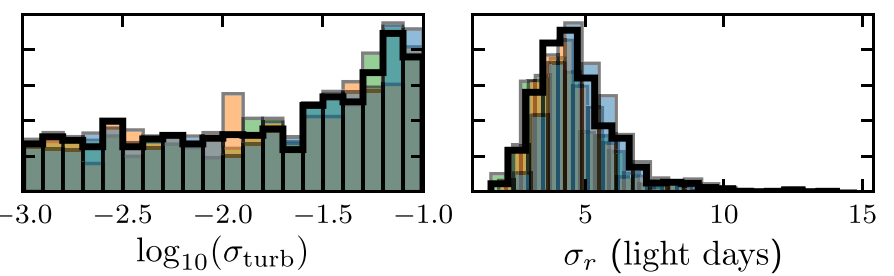

$\theta_{e}$ (degrees)

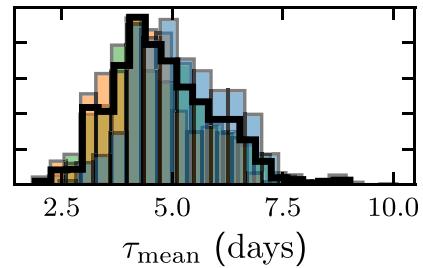

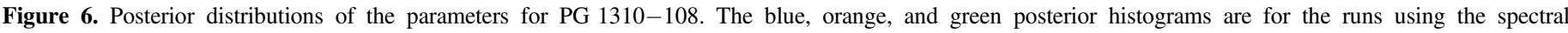
decompositions using the K10, BG92, and VC04 Fe II templates, respectively. The black line shows the combined posterior PDF.

suggest that the scale factor for this object, when using the line dispersion measured in the mean spectrum, should be $\log _{10}\left(f_{\text {mean }, \sigma}\right)=-0.20_{-0.21}^{+0.27} \quad\left(f=0.63_{-0.24}^{+0.54}\right)$, which is much smaller than the values that are typically used (e.g., $f_{\text {mean }, \sigma}=3.85 \pm 1.15$; Collin et al. 2006). The lower scale factor in this object illustrates the importance of calculating scale factors on an individual AGN basis. The use of a mean scale factor leads to underestimates and overestimates of $M_{\mathrm{BH}}$ in objects with higher and lower intrinsic $f$ values, respectively.

\section{2. $M r k 50$}

The BLR in Mrk 50 was previously modeled by Pancoast et al. (2012) using the same LAMP 2011 data and an earlier version of the model used in this paper. Their model did not include the parameters $\gamma$ or $\xi$, which help introduce asymmetries in the broad-line profile, and the dynamics component did not allow for macroturbulent velocities or the possibility of unbound inflowing or outflowing gas. We also include an additional narrow-line component in the model and leave the AGN redshift as a free parameter. The spectral decomposition they use is from Barth et al. (2011b), and it uses the BG92 Fe II template. In our analysis, we use the spectra found adopting both the K10 and BG92 templates, but since both are in very good agreement (Figure 2), we do not expect this to introduce any discrepancies in our measurements.

As discussed in Section 2.1, the VC04 Fe II template produced poor fits for some of the Mrk 50 spectra, so we chose to only use the results from the K10 and BG92 templates for our final analysis. For completeness, we include the results from the VCO4 template in Figure 7, but the combined posteriors shown by the black lines are computed using only the other two templates.

The model for Mrk 50 fits the shape of the $\mathrm{H} \beta$ emission line very well, as shown in Figure 4 . The large-scale fluctuations in the integrated $\mathrm{H} \beta$ flux are well captured, but the small peak in flux in the first few epochs is not recovered.

The radial distribution of $\mathrm{H} \beta$ emission in Mrk 50 is between Gaussian and exponential, with a shape parameter $\beta=0.87_{-0.10}^{+0.13}$. The radial distribution is shifted by a minimum radius $r_{\min }=$ $0.90_{-0.62}^{+0.81} \mathrm{lt}$-days, and it has a mean radius $r_{\text {mean }}=8.23_{-0.53}^{+0.54}$ lt-days and a radial width $\sigma_{r}=6.41_{-0.57}^{+0.69}$ lt-days. The mean lag is slightly smaller than this with $\tau_{\text {mean }}=7.43_{-0.41}^{+0.45}$ days, which is again smaller than the cross-correlation measurement $\tau_{\text {cen }}=$ $8.66_{-1.51}^{+1.63}$ days from A. J. Barth et al. (2018, in preparation), but is consistent to within the uncertainties. The opening and inclination angles are well constrained and prefer a slightly thick disk geometry, oriented close to face-on $\left(\theta_{o}=14.1_{-3.7}^{+4.8}, \theta_{i}=\right.$ $19.8_{-5.4}^{+6.0}$ degrees). An example of this geometry is shown in Figure 3. There is a slight preference for the emission to be concentrated near the faces of the disk, with $\gamma=3.9_{-1.3}^{+0.8}$, but uniform emission throughout the disk is not ruled out. The disk midplane is mostly opaque $\left(\xi=0.15_{-0.10}^{+0.16}\right)$ and the relative strength of the emission from the near and far side of the BLR is equal $\left(\kappa=-0.03_{-0.10}^{+0.13}\right)$.

Our geometric model results are generally in good agreement with those of Pancoast et al. (2012). The largest discrepancy is in the opening and inclination angles, where our results show slightly larger values for both angles. We do find a disk midplane that is mostly opaque, which was not possible in the earlier version of the model. The $2 \mathrm{D}$ posterior samples for these 

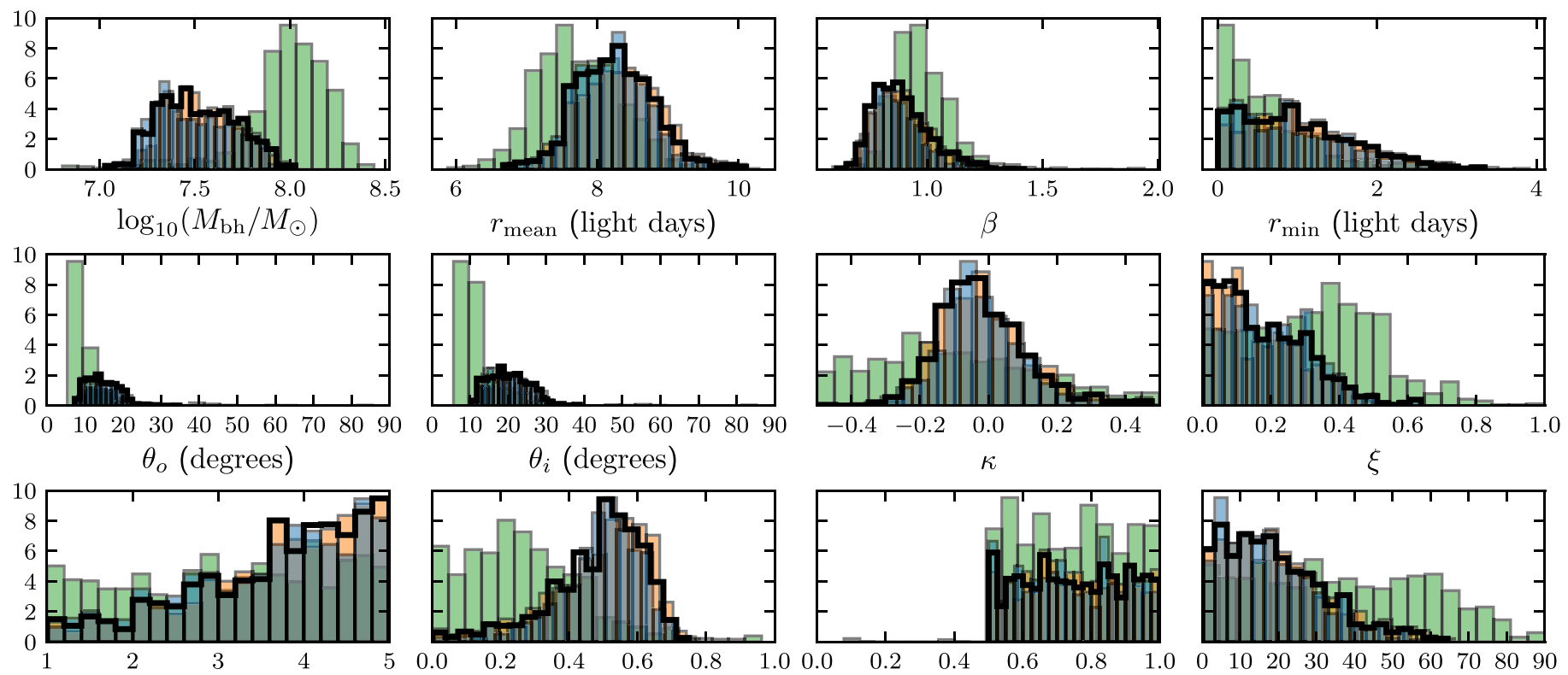
$\theta_{i}$ (degrees)
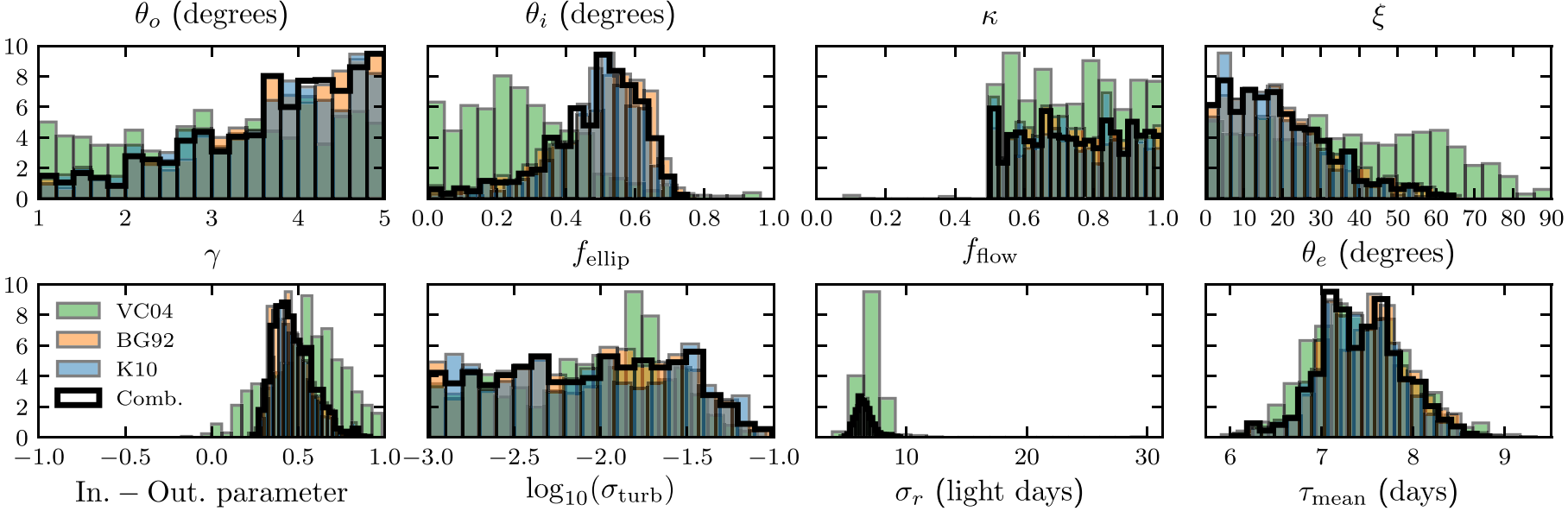

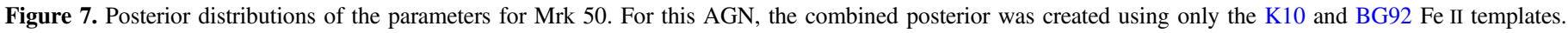

parameters show that smaller opening and inclination angles are preferred for higher values of $\xi$ (transparent midplane), so it is possible that this new flexibility is the main cause of the discrepancy.

Dynamically, the model of Mrk 50 prefers solutions in which half of the particles are on near-circular elliptical orbits $\left(f_{\text {ellip }}=0.51_{-0.15}^{+0.10}\right)$. The remaining particles have velocities drawn from a distribution in $v_{r}-v_{\phi}$ space with an outflowing radial component $\left(f_{\text {flow }}=0.75_{-0.17}^{+0.17}\right)$. The center of this distribution is rotated $\theta_{e}=17_{-12}^{+15}$ degrees from the radially outflowing escape velocity toward the circular velocity. The contribution of macroturbulent velocities is minimal, with $\sigma_{\text {turb }}=0.009_{-0.007}^{+0.022}$.

We find the black hole mass to be $\log _{10}\left(M_{\mathrm{BH}} / M_{\odot}\right)=$ $7.50_{-0.18}^{+0.25}$. This is consistent with the Pancoast et al. (2012) measurement of $7.57_{-0.27}^{+0.44}$, despite the different models. This is reassuring but also perhaps not surprising, given that we do not find a significant inflow or outflow component that our model would be able to better describe than the previous version.

\section{3. $M r k 141$}

The data set for Mrk 141 is of relatively low quality owing to many spectroscopic observing nights lost to poor weather. The integrated $\mathrm{H} \beta$ light curve is relatively short and there are not many strong variability features, but there is one large increase and decrease in flux over the course of the campaign. The models are able to fit this overall feature, but do not fit the smaller fluctuations on scales of a few days. The models are able to fit the shape of the $\mathrm{H} \beta$ profile very well.
The posterior PDFs from runs using all three Fe II templates agree very well for most parameters, with the largest discrepancy coming from the parameter $\kappa$. The radial distribution of the BLR in Mrk 141 is roughly exponential, with shape parameter $\beta=1.02_{-0.17}^{+0.16}$, and is shifted from the origin by $r_{\min }=2.08_{-0.85}^{+0.89}$ lt-days. The mean radius is $r_{\text {mean }}=$ $8.1_{-1.7}^{+1.8}$ lt-days, and the radial width of the distribution is $\sigma_{r}=$ $6.1_{-1.6}^{+2.4}$ lt-days. The mean lag is very similar to $c \times r_{\text {mean }}$, with $\tau_{\text {mean }}=7.5_{-1.6}^{+1.7}$ days. This value is consistent with the crosscorrelation measurement $\tau_{\text {cen }}=5.63_{-1.65}^{+8.27}$ days.

The opening and inclination angles indicate a thick disk $\left(\theta_{o}=15.3_{-2.5}^{+3.9}\right.$ degrees) inclined $\theta_{i}=26.0_{-4.3}^{+6.0}$ degrees relative to the observer. As with Mrk 50, there is a small preference for $\mathrm{H} \beta$ emission to be concentrated near the faces of the disk, but uniform emission is not ruled out $\left(\gamma=3.9_{-1.3}^{+0.8}\right)$. The midplane of the disk is opaque $(\xi<0.071)$. All three posterior PDFs generated using each Fe II template indicated a preference for emission from the far side of the BLR, but the result is slightly more pronounced using the K10 template results. In the combined posterior, $\kappa=-0.224_{-0.078}^{+0.059}$.

Dynamically, $<20 \%$ of the $\mathrm{H} \beta$-emitting BLR is on nearcircular elliptical orbits $\left(f_{\text {ellip }}=0.104_{-0.068}^{+0.082}\right)$. The remainder have velocities drawn from a distribution around the outflowing escape velocity, rotated $\theta_{e}=14_{-10}^{+16}$ degrees toward the circular velocity in the $v_{r}-v_{\phi}$ plane. Macroturbulent velocities are not significant in Mrk 141 with $\sigma_{\text {turb }}=0.005_{-0.004}^{+0.011}$.

We find the black hole mass in Mrk 141 to be $\log _{10}\left(M_{\mathrm{BH}} / M_{\odot}\right)=7.46_{-0.21}^{+0.15}$. Previous measurements of the black hole mass have been made using the BLR radiusluminosity relation and the FWHM of the $\mathrm{H} \beta$ line, which find 

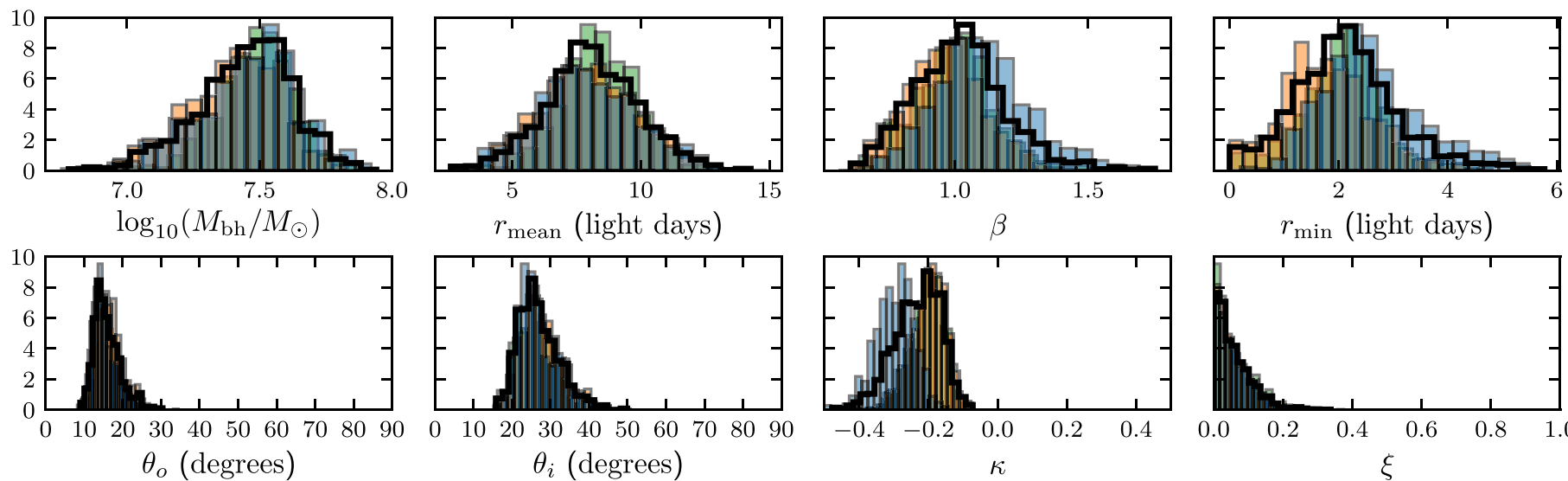

$r_{\text {mean }}$ (light days)

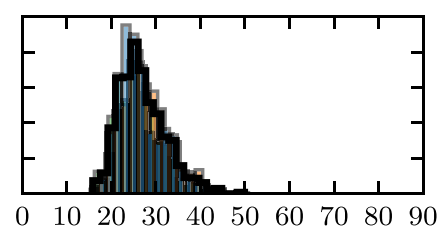

$\beta$
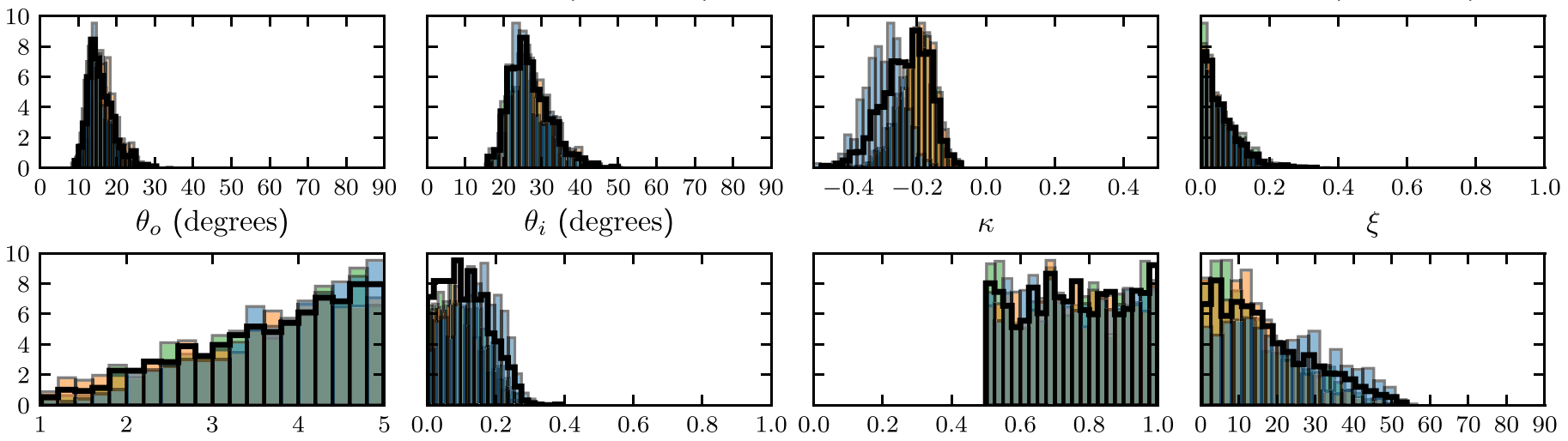
$\theta_{i}$ (degrees)
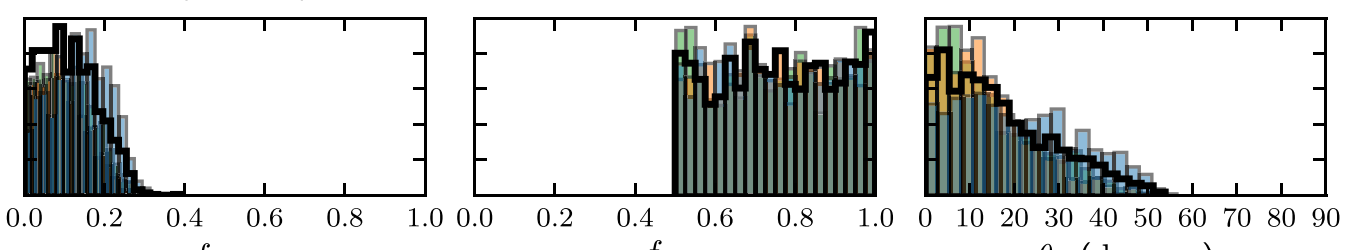

$\gamma$
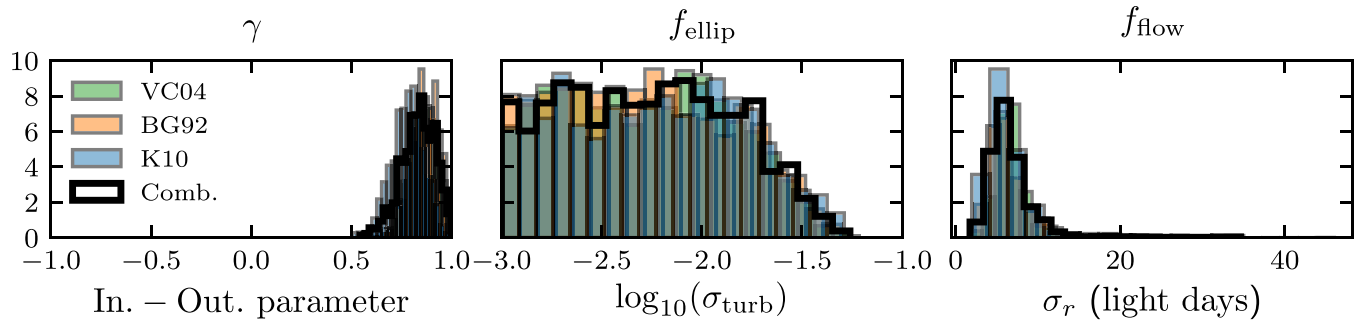

$\theta_{e}$ (degrees)

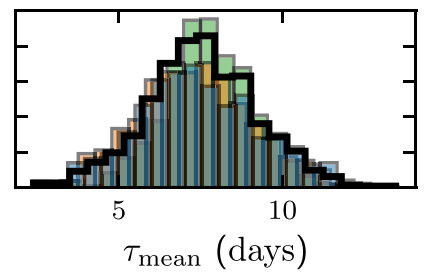

Figure 8. Posterior distributions of the parameters for Mrk 141.

$\log _{10}\left(M_{\mathrm{BH}} / M_{\odot}\right)=7.53$ (Castelló-Mor et al. 2017) and $7.85(\mathrm{Li}$ et al. 2008). These studies do not report uncertainties, but assuming a typical uncertainty of $0.4 \mathrm{dex}$ arising from the scatter in the $r-L$ relation and the uncertainty in the scale factor used, our result is consistent with both measurements.

\section{4. $M r k 279$}

The $\mathrm{H} \beta$ line profile for Mrk 279 is modeled very well, with only a slight discrepancy at the red side of the core of the line. The large timescale variations of the integrated $\mathrm{H} \beta$ line flux are well captured, but the model is unable to reproduce the smaller fluctuations on the order of days.

The Mrk $279 \mathrm{H} \beta$-emitting region has a radial profile that is poorly determined, with anything from a narrow Gaussian to a steeper than exponential profile being allowed $\left(\beta=1.04_{-0.64}^{+0.71}\right)$. The minimum radius is found to be large, with $r_{\min }=$ $9.2_{-2.9}^{+2.1}$ lt-days. The $2 \mathrm{D}$ posterior distributions of these two parameters show that smaller values of the minimum radius ( $<5$ lt-days) are allowed when $\beta<0.5$ (narrow Gaussian), but for wider Gaussian and steep exponential profiles, the minimum radius is robustly determined.

The opening angle and inclination angle for Mrk 279 are $\theta_{o}=41.0_{-4.1}^{+4.3}$ degrees and $\theta_{i}=29.1_{-3.4}^{+3.4}$ degrees, respectively, indicating a thick disk that is slightly inclined relative to the observer. Based on the full posterior PDF for $\gamma$, there is no preference for emission to either be concentrated near the faces of the disk or for it to be uniform throughout. There is a very strong preference for emission from the far side of the BLR $(\kappa<-0.46)$, and the midplane of the disk is fully opaque $(\xi<0.063)$. This shows up clearly in the geometric model
(Figure 3) in that there are very few points visible on the bottom-left half of the edge-on view, and the points that are farther from the observer are larger, representing the relative strength of the emission.

In Mrk 279, models with almost no particles on near-circular orbits are preferred $\left(f_{\text {ellip }}<0.081\right)$. Instead, velocities are drawn from a distribution rotated $\theta_{e}=21.7_{-6.0}^{+7.8}$ degrees from the radially outflowing escape velocity toward the circular velocity. The upward-angled outflow signature discussed in Section 4.3 is prominent in the Mrk 279 transfer function (Figure 5) and is the strongest for the whole LAMP 2011 sample. There is no indication of a significant contribution from macroturbulent velocities ( $\sigma_{\text {turb }}=0.0037_{-0.0023}^{+0.0065}$ ).

We find a black hole mass of $\log _{10}\left(M_{\mathrm{BH}} / M_{\odot}\right)=7.58_{-0.08}^{+0.08}$ in Mrk 279. This object was spectroscopically monitored in 1988, when Maoz et al. (1990) found the size of the $\mathrm{H} \beta$-emitting BLR to be $11 \pm 3$ lt-days. Santos-Lleó et al. (2001) observed Mrk 279 from 1996 to 1997 and measured an $\mathrm{H} \beta$ time lag of $\tau=16_{-5.6}^{+5.3}$ days. Peterson et al. (2004) later re-analyzed both data sets and, assuming a value of 5.5 for $f_{\text {rms }, \sigma}$, measured the black hole mass to be $M_{\mathrm{BH}} /\left(10^{6} M_{\odot}\right)=34.9 \pm 9.2$ $\left[\log _{10}\left(M_{\mathrm{BH}} / M_{\odot}\right)=7.54_{-0.13}^{+0.10}\right]$. Our results for the size, time lag, and black hole mass are consistent with all of these results. We note that the true BLR size and $\mathrm{H} \beta$ time lag may have changed between our three campaigns owing to changes in the AGN continuum, but the black hole mass should remain the same. Mrk 279 was also analyzed by Castelló-Mor et al. (2017) using the radius-luminosity relationship as a BLR size estimator, and they found $\log _{10}\left(M_{\mathrm{BH}} / M_{\odot}\right)=7.97$. As in the case of Mrk 141, our results are consistent with this 

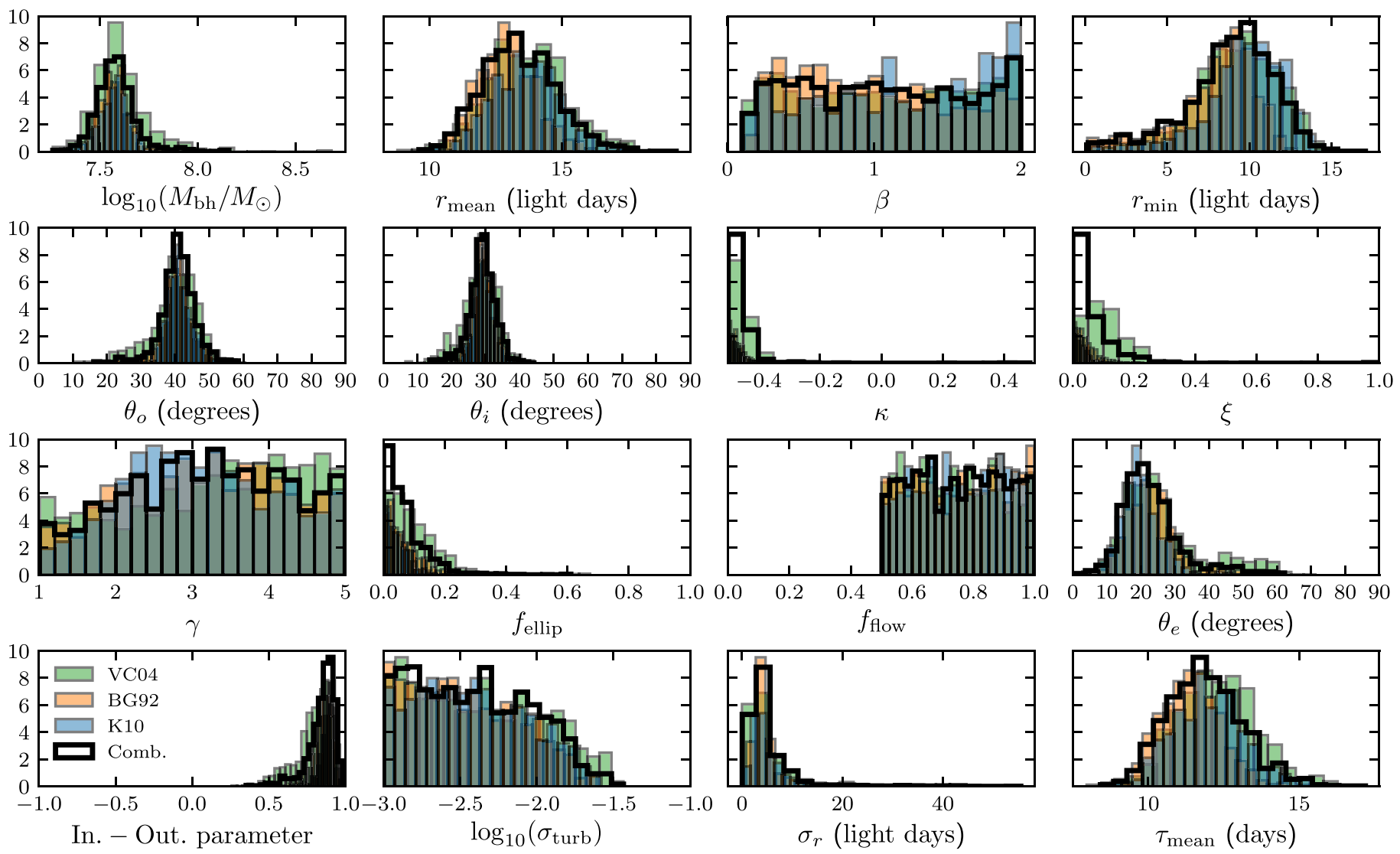

$\theta_{e}$ (degrees)

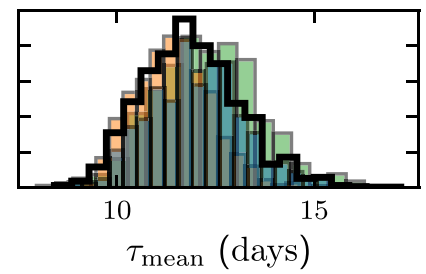

Figure 9. Posterior distributions of the parameters for Mrk 279.

measurement when realistic uncertainties are assumed for the previous estimates.

\section{5. $M r k 1511$}

Both the $\mathrm{H} \beta$ emission-line shape and the variability in the integrated $\mathrm{H} \beta$ flux are very well fit by the models for Mrk 1511. However, the posterior PDFs in Figure 10 indicate that the $\mathrm{H} \beta$ spectra produced using the three Fe II templates give somewhat different modeling results. The right panels of Figure 2 show that there is a discrepancy between the three $\mathrm{H} \beta$ profiles that is on the order of the size of the flux uncertainty. This is likely a result of the strong Fe II contribution to the AGN spectrum, shown by the green line in the left panel. Since the K10 template is made up of five individual components whose strengths are given by five free parameters, it has more flexibility to fit asymmetries in the Fe II emission than the other templates. This can result in asymmetries in the resulting $\mathrm{H} \beta$ spectrum. Since the model results are still consistent with each other, we choose to combine them, the result of which is larger parameter uncertainties.

The $\mathrm{H} \beta$-emitting BLR in Mrk 1511 has a radial profile that is between Gaussian and exponential $\left(\beta=0.61_{-0.10}^{+0.14}\right)$ and is shifted from the central ionizing source by $r_{\min }=0.72_{-0.53}^{+0.96} \mathrm{lt}$ days. The mean radius is $r_{\text {mean }}=5.52_{-0.50}^{+0.55} \mathrm{lt}$-days, and the radial thickness is $\sigma_{r}=2.85_{-0.40}^{+0.45} \mathrm{lt}$-days. The mean time lag is $\tau_{\text {mean }}=5.94_{-0.46}^{+0.45}$ days, consistent with the cross-correlation lag measurement of $\tau_{\text {cen }}=5.44_{-0.67}^{+0.74}$ days. The structure of the BLR is best described by a thick disk with opening angle $\theta_{o}=$ $36_{-10}^{+9}$ degrees that is inclined by $\theta_{i}=19.3_{-4.7}^{+5.7}$ degrees relative to the observer. The $\mathrm{H} \beta$ emission is mostly uniform throughout the disk, as opposed to being concentrated near the faces $(\gamma<1.9)$. There is a strong preference for emission from the far side of the BLR $(\kappa<-0.39)$, and the disk midplane is transparent $\left(\xi=0.85_{-0.19}^{+0.09}\right)$.

The largest discrepancy in the results from the three runs comes from the dynamical component of the model. The results from using the $\mathrm{VCO} 4 \mathrm{Fe}$ II template indicates that half of the BLR is in near-circular orbits while the other half is in nearradial infall. The results from using the K10 and BG92 templates suggest that closer to three-fourths of the BLR is in near-circular orbits. The remaining one-fourth is in close-toradial infall, though radial outflow is not fully ruled out. Combining all three posteriors, we find $f_{\text {ellip }}=0.62_{-0.14}^{+0.16}$ of the particles on near-circular orbits, with the remaining particles close to radial infall $\left(f_{\text {flow }}=0.27_{-0.18}^{+0.19}, \theta_{e}=9_{-6}^{+14}\right.$ degrees $)$. Of all the objects in the LAMP 2011 sample, macroturbulent velocities have the highest effect on the BLR dynamics in Mrk 1511, with $\sigma_{\text {turb }}>0.029$. The posterior PDF shows that this value is approaching its prior bound of 0.1 , so the contribution may actually be higher. We find the black hole mass in Mrk 1511 to be $\log _{10}\left(M_{\mathrm{BH}} / M_{\odot}\right)=7.11_{-0.17}^{+0.20}$.

\section{6. $N G C 4593$}

The models for NGC 4593 fit the observed $\mathrm{H} \beta$ emission-line shape very well. Additionally, the models are able to recover almost all of the variation in the $\mathrm{H} \beta$ light curve, including the short-timescale variations. As with Mrk 1511, the results from using different Fe II templates show disagreement in some parameters. Looking at the spectral decomposition for NGC 4593, there is a significant difference between the 

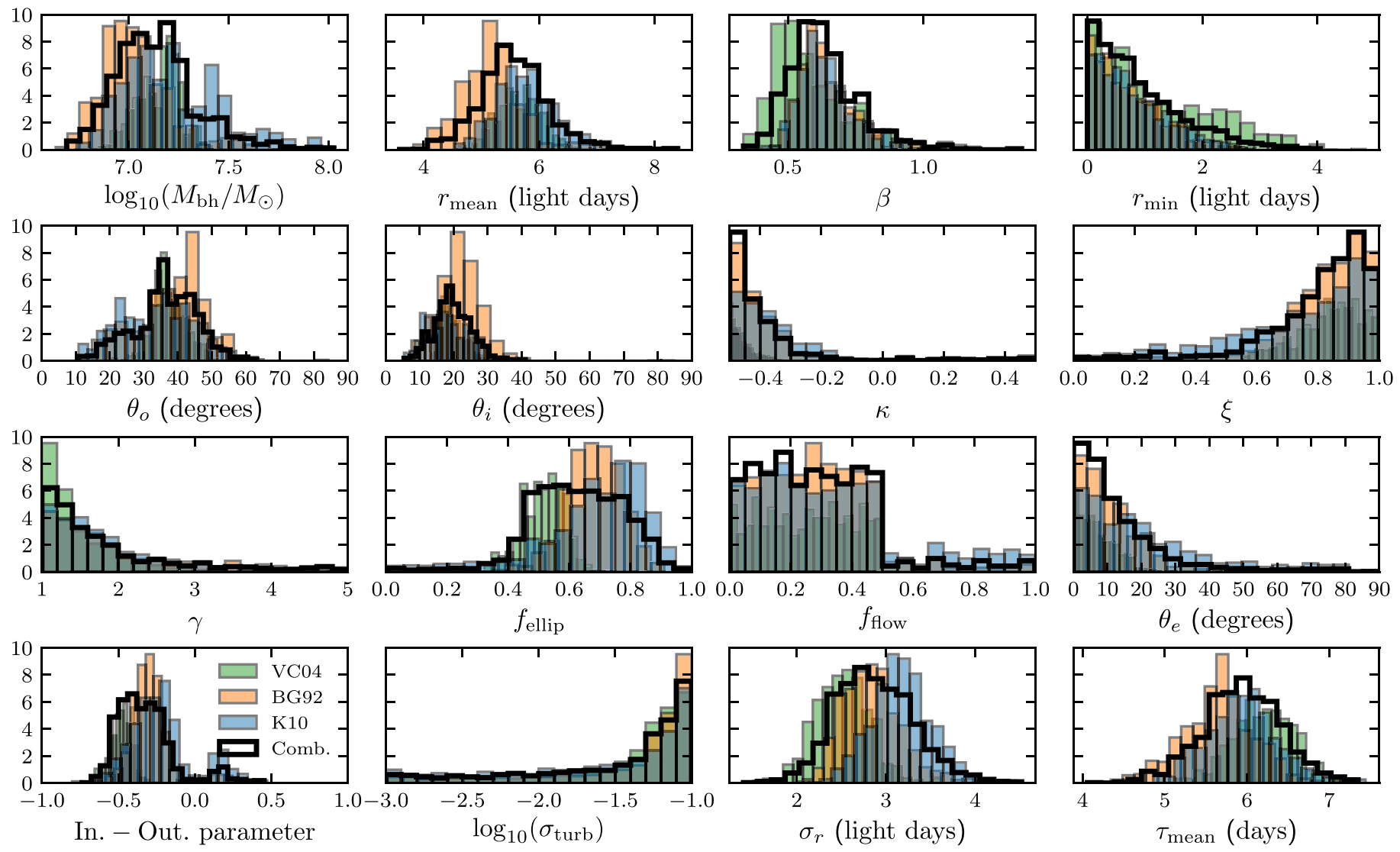

Figure 10. Posterior distributions of the parameters for Mrk 1511.

$\mathrm{H} \beta$ profiles produced using the three templates, especially in the wings of the line, owing to the strong Fe II emission in this object.

The results from using the K10 and BG92 Fe II templates show that the radial profile of the NGC 4593 BLR is between Gaussian and exponential, with the minimum radius poorly constrained. When the VC04 template is used, the Gamma function shape parameter is poorly constrained, but the minimum radius is found to be about 1.8 lt-days. Combining the posteriors, we find a radial profile that is near exponential $\left(\beta=1.01_{-0.25}^{+0.61}\right)$ and is shifted from the central ionizing source by $r_{\min }=1.00_{-0.65}^{+0.80}$ lt-days. The mean radius is $r_{\text {mean }}=$ $3.41_{-0.55}^{+0.51} \mathrm{lt}$-days, and the radial thickness is $\sigma_{r}=2.41_{-0.49}^{+0.72} \mathrm{lt}$ days. The mean time lag is $\tau_{\text {mean }}=3.29_{-0.40}^{+0.48}$ days, which is consistent with the cross-correlation lag measurement of $\tau_{\text {cen }}=3.54_{-0.82}^{+0.76}$ days. The structure is best described by a thick disk with opening angle $\theta_{o}=43_{-19}^{+22}$ degrees that is inclined by $\theta_{i}=32_{-10}^{+19}$ degrees relative to the observer. The $\mathrm{H} \beta$ emission is mostly uniform throughout the disk $(\gamma<3.0)$, there is a preference for emission from the far side of the BLR $\left(\kappa=-0.25_{-0.22}^{+0.28}\right)$, and the disk midplane is between transparent and opaque $\left(\xi=0.41_{-0.30}^{+0.27}\right)$.

Dynamically, the results from the three runs show disagreement in the amounts of inflowing or outflowing gas. The results from using the K10 and BG92 Fe II templates are in agreement, with three-fourths of the particles on near-circular orbits, and the remainder on radially outflowing trajectories. Using the VC04 template, only one-fourth of the orbits are inferred to be near-circular, with the remainder in near-radial inflow. Outflowing trajectories, however, are not fully ruled out. All three runs give the same result that macroturbulent velocities may be important for the dynamics of NGC $4593\left(\sigma_{\text {turb }}>0.014\right)$. As with Mrk 1511, this parameter is approaching its prior bound of 0.1 , meaning the contribution could be larger.

Despite the differences in inferred BLR structure and dynamics, all three runs converge to the same black hole mass of $\log _{10}\left(M_{\mathrm{BH}} / M_{\odot}\right)=6.65_{-0.15}^{+0.27}$.

\subsection{Zw 229-015}

The spectroscopic monitoring for $\mathrm{Zw} 229-015$ also suffered from losses owing to poor weather, having the fewest spectroscopic observations in the sample. The large-scale variability features are driven by the final three data points, with some shorter timescale variability around the peak. The models are able to recover the variability on both long and short timescales. The $\mathrm{H} \beta$ line profile's asymmetric shape is also very well modeled.

The radial profile of the BLR in $\mathrm{Zw} 229-015$ is steeper than exponential $\left(\beta=1.36_{-0.30}^{+0.31}\right)$ and is shifted from the central ionizing source by $r_{\min }=2.19_{-0.61}^{+0.72}$ lt-days. The mean radius is $r_{\text {mean }}=6.94_{-0.97}^{+0.99}$ lt-days, and the radial thickness is of similar size, $\sigma_{r}=6.3_{-1.5}^{+1.9}$ lt-days. The mean lag is inferred to be $\tau_{\text {mean }}=6.47_{-0.87}^{+0.90}$ days, which is consistent with the crosscorrelation measurement of $\tau_{\text {cen }}=5.90_{-2.40}^{+7.61}$ days. $\mathrm{Zw} 229$ -015 was also monitored at Lick Observatory from 2010 June to December, and Barth et al. (2011a) measured an $\mathrm{H} \beta$ lag of $\tau_{\text {cen }}=3.86_{-0.90}^{+0.69}$ days using these data. The structure of the BLR is well constrained to be a thick disk $\left(\theta_{o}=33.5_{-6.2}^{+6.4}\right.$ degrees), inclined $\theta_{i}=32.9_{-5.2}^{+6.1}$ degrees relative to the observer. There is little preference for the emission to be 

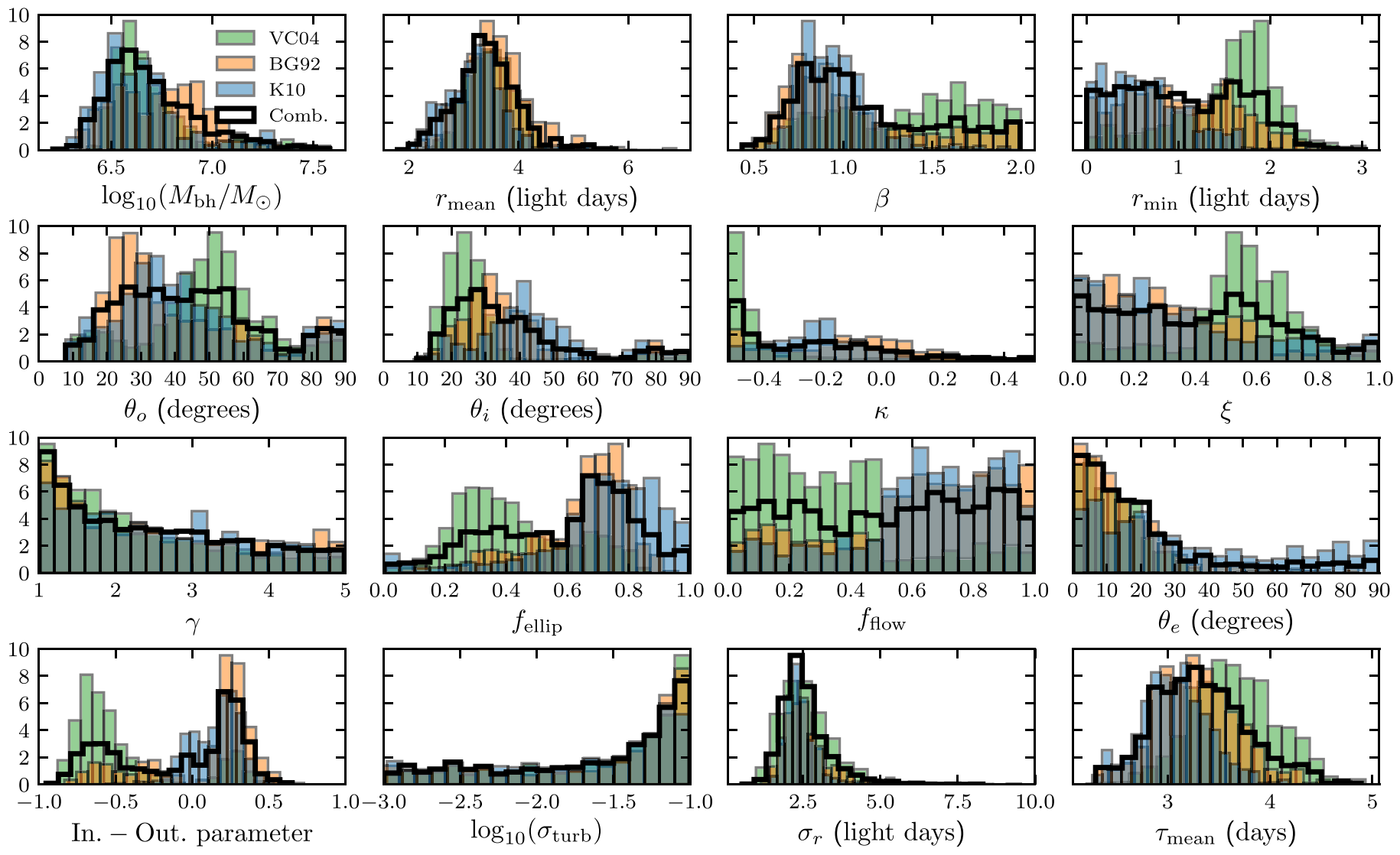

Figure 11. Posterior distributions of the parameters for NGC 4593.

either concentrated near the faces of the disk or distributed uniformly throughout the disk. The emission comes mostly from the far side of the BLR $\left(\kappa=-0.417_{-0.054}^{+0.065}\right)$, and the midplane of the disk is fully opaque $(\xi<0.080)$.

Dynamically, models in which almost all particles are on outflowing trajectories are preferred. The fraction of the BLR that is on near-circular orbits is $f_{\text {ellip }}<0.15$. The remaining particles have velocities drawn from a distribution whose center is rotated $\theta_{e}=10.9_{-7.2}^{+9.0}$ degrees from the radially outflowing escape velocity toward the circular velocity in the $v_{r}-v_{\phi}$ plane. Barth et al. (2011a) split the $\mathrm{H} \beta$ profile of $\mathrm{Zw} 229-015$ into six bins to make velocity-resolved reverberation mapping measurements. Qualitatively, they find results that are consistent with Keplerian motions, although they do not state conclusive evidence owing to the large error bars on the measurements. It is inconclusive from the results whether macroturbulent velocities contribute significantly to the BLR dynamics $\left(\sigma_{\text {turb }}=0.024_{-0.021}^{+0.048}\right)$. This parameter is approaching its prior bound of 0.1 , so it is possible that the contribution is higher.

We find the black hole mass in $\mathrm{Zw} 229-015$ to be $\log _{10}\left(M_{\mathrm{BH}} / M_{\odot}\right)=6.94_{-0.14}^{+0.14}$. This is consistent with the value of $\log _{10}\left(M_{\mathrm{BH}} / M_{\odot}\right)=7.00_{-0.12}^{+0.08}$ from Barth et al. (2011a), even though the measured lag changed between the two campaigns. This is reassuring since, while the BLR size (and hence lag) may change on these timescales due to changes in the ionizing continuum, the black hole mass should remain the same. The consistency in measurements across the two epochs serves as a test of the two methods.

\section{Discussion}

\subsection{Overall Properties of the LAMP 2011 BLRs}

The results from this analysis paint a fairly uniform picture for the geometric structure of the $\mathrm{H} \beta$-emitting BLR in the AGN in this sample. We note, however, that this sample spans a limited range in luminosity and black hole mass, so BLR geometries may differ for objects outside of this parameter space. We find the BLR in all objects to be thick disks that are viewed close to face-on. The radial distribution of particles is typically between Gaussian and exponential, when well determined. There is a preference for emission from the far side of the BLR in all objects, except for Mrk 50, whose results allow for the possibility of preferential emission from the near side. Emission from the far side is what one expects based on photoionization model predictions that $\mathrm{H} \beta$ is mostly re-emitted back toward the ionizing source (Ferland et al. 1992; O'Brien et al. 1994). Most objects allow for the possibility of either uniform emission throughout the disk or concentrated emission near the faces of the disk. Mrk 1511, the only object in which this parameter is well constrained, shows a strong preference for uniform emission. Finally, the parameter determining the transparency of the disk midplane varies across our sample.

Dynamically, we find more variety in our sample. The contribution of macroturbulent velocities is unconstrained or negligible in most objects, but Mrk 1511, NGC 4593, and Zw 229-015 show a possible significant contribution with $\sigma_{\text {turb }}$ approaching its upper prior bound of 0.1 . Three of the objects (Mrk 50, Mrk 1511, and NGC 4593) are found to have more than half of the particles on near-circular orbits, and the 

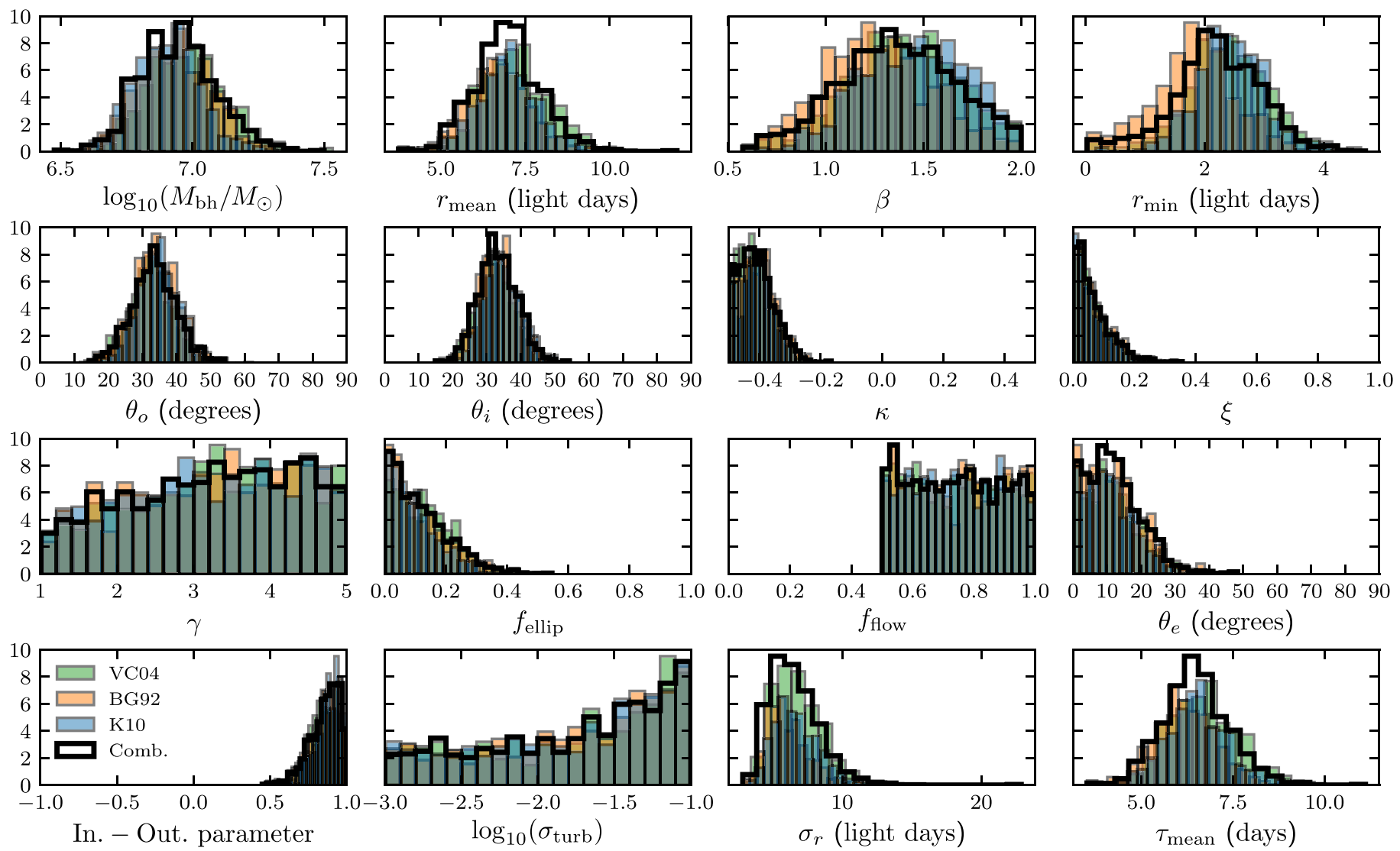

Figure 12. Posterior distributions of the parameters for $\mathrm{Zw} 229-015$.

rest have almost all particles on either inflowing or outflowing trajectories. For every object except Mrk 1511, the particles that are not on near-circular orbits are on outflowing trajectories. This is in contrast to the results from P14 and G17, both of which find mostly near-circular orbits, inflowing orbits, or a combination of the two. To our knowledge, outflow in the $\mathrm{H} \beta$-emitting BLR has only been observed in three other cases: NGC 3227 (Denney et al. 2009b), Mrk 142 (Du et al. 2016), and MCG +06-26-012 (Du et al. 2016). The model used in this paper is not able to constrain the detailed dynamics of the outflow. If the outflow is launched and then the gas is left to move under the influence of the black hole's gravity, then the assumption that the black hole's gravity dominates BLR motions holds. However, significant outward forces due to (for example) radiation pressure might lead us to underestimate the mass (Marconi et al. 2008, 2009; Netzer 2009; Netzer \& Marziani 2010). A more detailed analysis of the dynamics will be necessary to fully understand how outflows affect black hole mass measurements.

\subsection{Scale Factor $\mathrm{f}$}

The scale factor $f$ gives the relationship between the virial product $\left(M_{\mathrm{vir}}=c \tau v^{2} / G\right)$ and the black hole mass $\left(M_{\mathrm{BH}}=f M_{\mathrm{vir}}\right)$. This value depends on the structure and physical orientation of the BLR as well as the dynamics and emitting properties of the gas that can affect the asymmetry of the broadline profile. We do not expect all of these properties to be the same for every AGN, so each AGN has its own conversion factor between the virial product and the black hole mass. However, since traditional reverberation mapping cannot recover individual scale factors, an average value is typically used. This is measured by finding the value that puts AGN black hole mass measurements in alignment with the $M_{\mathrm{BH}}-\sigma_{*}$ relation for quiescent galaxies. Results from these analyses range from $f_{\text {rms, } \sigma}=2.8_{-0.5}^{+0.7}$ (i.e., $\log _{10}\left(f_{\mathrm{rms}, \sigma}\right)=0.44_{-0.09}^{+0.10}$; Graham et al. 2011) to $f_{\mathrm{rms}, \sigma}=$ $5.9_{-1.5}^{+2.1}$ (i.e., $\log _{10}\left(f_{\mathrm{rms}, \sigma}\right)=0.77 \pm 0.13$; Woo et al. 2013), though these measurements are generally consistent to within the uncertainties.

Using the direct modeling approach, we can calculate the scale factor $f$ for individual AGNs by combining the black hole masses inferred by our model with line width and BLR size measurements. In the following sections, we use BLR size measurements based on $r=c \tau$, where the time delay is the cross-correlation measurement $\tau_{\text {cen }}$ from A. J. Barth et al. (2018, in preparation). The line widths consist of three measurements: the FWHM measured in the mean spectrum, the line dispersion measured in the mean spectrum, and the line dispersion measured in the rms spectrum. All line widths for the individual sources presented here come from Barth et al. (2015).

To propagate uncertainty, we first assume Gaussian errors in the line width and $\tau$ measurements, with standard deviations given in the respective papers. We take random draws from these distributions to generate a sample of virial product values of the same size as the black hole mass posterior sample. The scale-factor posterior distribution is then calculated by dividing the black hole mass by the virial product distributions.

\subsubsection{Mean $\mathrm{f}$ for the LAMP 2011 Sample}

Here, we examine the mean scale factor for the LAMP 2011 sample as well as the mean scale factor for the sample of all 
Table 3

BLR Model Parameter Values

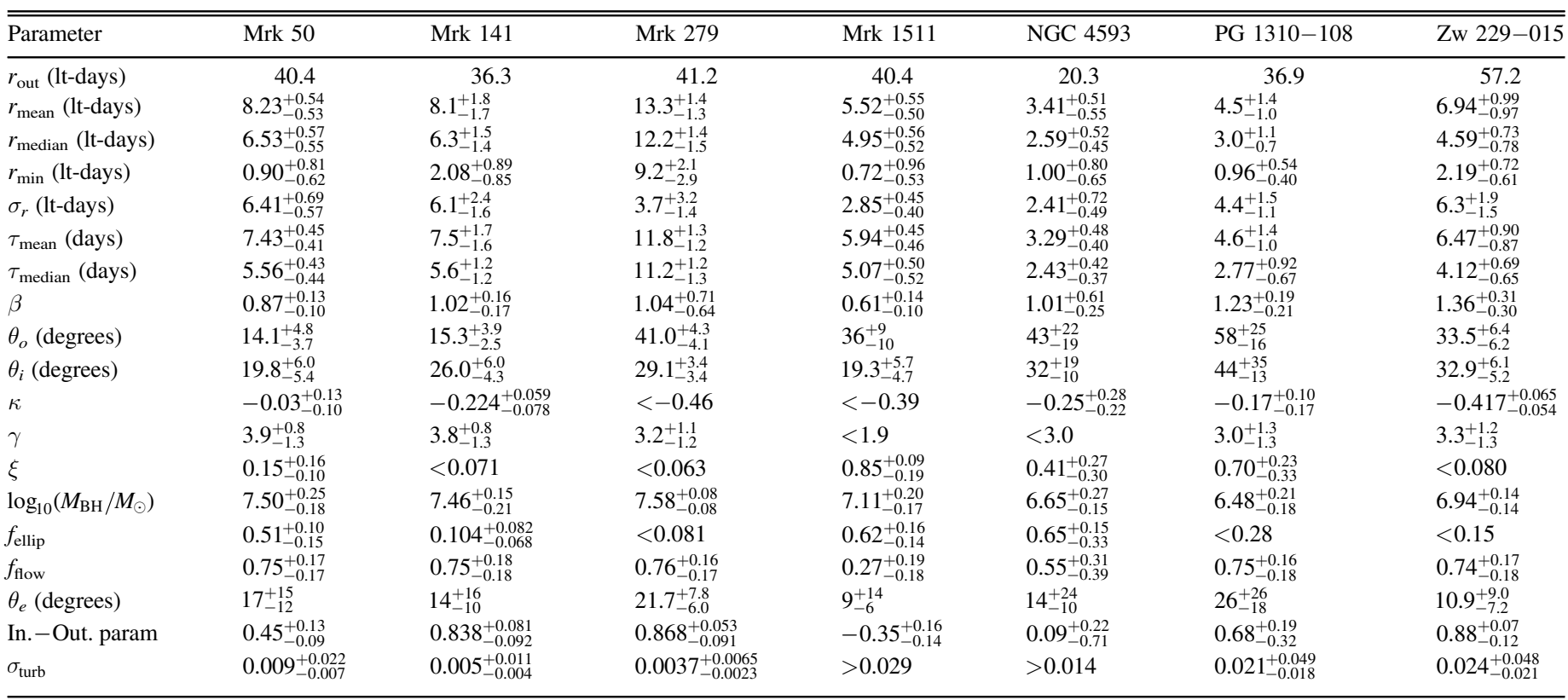

Note. Median values and $68 \%$ confidence intervals for the main BLR geometry and dynamic model parameters. Upper and lower $68 \%$ confidence limits are given when the posterior PDF is one-sided. Note that $r_{\text {out }}$ is a fixed parameter, so we do not include uncertainties.
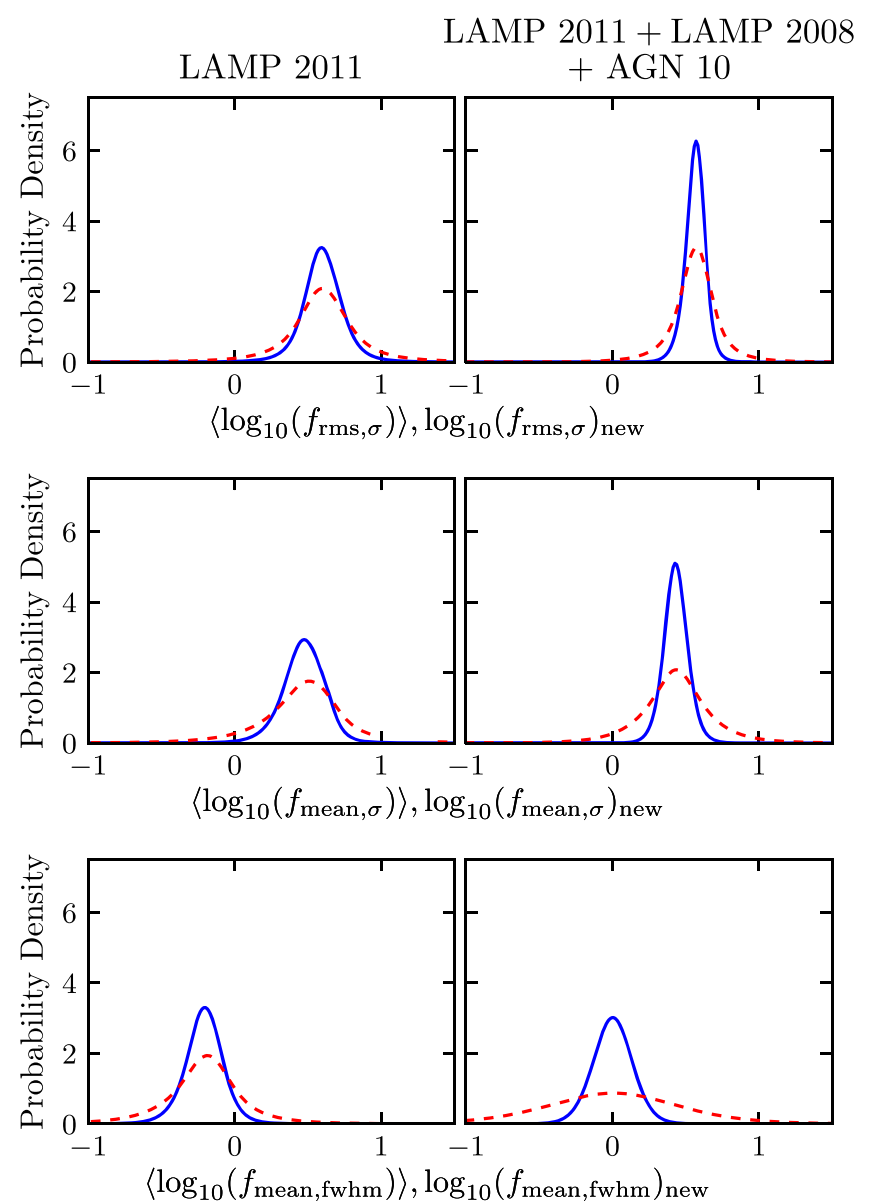

Figure 13. Mean scale-factor posterior distribution (blue, solid) and the posterior predictive distribution for new scale-factor measurements (red, dashed). The left-hand panels show the results for the AGNs discussed in this paper. The right panels show the results when the sample from this paper is combined with the samples from P14 and G17.
AGNs analyzed using the direct modeling method in this paper. We model the distribution of scale factors as Gaussian with mean $\log _{10} \bar{f}$ and standard deviation $\sigma_{\log _{10} f}$ given by the dispersion in the individual values of $\log _{10} f$. The likelihood for different pairs of $\left(\log _{10} \bar{f}, \sigma_{\log _{10} f}\right)$ is calculated using the full posterior PDFs for each AGN. From this analysis, we can determine both $\log _{10} \bar{f}$ and $\sigma_{\log _{10} f}$ as well as uncertainties in each value. We also calculate the posterior predictive distribution for our sample, which marginalizes over the uncertainty in $\log _{10} \bar{f}$ and $\sigma_{\log _{10} f}$ :

$$
p\left(f_{\text {pred }} \mid \boldsymbol{X}\right)=\int_{\theta} p\left(f_{\text {pred }} \mid \theta, \boldsymbol{X}\right) p(\theta \mid \boldsymbol{X}) d \theta
$$

where $\boldsymbol{X}$ is the sample of measurements and $\theta=$ $\left(\log _{10} \bar{f}, \sigma_{\log _{10} f}\right)$. This is the distribution from which future scale-factor measurements are drawn and is wider than the distribution of our measured scale factors owing to the uncertainty on $\log _{10} \bar{f}$ and $\sigma_{\log _{10} f}$. The posterior distributions and posterior predictive distributions are given in Figure 13.

The same scale-factor analysis was performed by P14 (five objects) and G17 (four objects). Here, we combine our sample of seven objects with those results to expand the sample to 16 objects. The results for the LAMP 2011 sample and the combined sample of 16 objects are summarized in Table 4 . We find that our results for $\log _{10}\left(\bar{f}_{\text {rms }, \sigma}\right)$ both in the LAMP 2011 sample and the combined sample are consistent with the results of previous studies (e.g., Onken et al. 2004; Woo et al. 2010; Graham et al. 2011; Grier et al. 2013a; Woo et al. 2013) to within the quoted uncertainties. However, we note that PG 1310-108, which has the smallest value for both $\log _{10}\left(f_{\text {mean }, \sigma}\right)$ and $\log _{10}\left(f_{\text {mean,FWHM }}\right)$, does not have a $\log _{10}\left(f_{\text {rms }, \sigma}\right)$ measurement, owing to the low signal-to-noise ratio in the rms spectrum. If the $\log _{10}\left(f_{\mathrm{rms}, \sigma}\right)$ for this object has a similarly small value, this could reduce our mean value to be inconsistent with some of the higher measurements. 
Table 4

Summary of Scale Factors

\begin{tabular}{llrcc}
\hline \hline & & Mean, FWHM & Mean, $\sigma$ & rms, $\sigma$ \\
\hline \multirow{3}{*}{ L11 } & $\log _{10}(\bar{f})$ & $-0.21 \pm 0.14$ & $0.47 \pm 0.15$ & $0.60 \pm 0.16$ \\
& $\sigma_{\log _{10} f}$ & $0.23 \pm 0.16$ & $0.25 \pm 0.17$ & $0.22 \pm 0.19$ \\
& $\log _{10}(f)_{\text {pred }}$ & $-0.21 \pm 0.31$ & $0.47 \pm 0.34$ & $0.60 \pm 0.32$ \\
\hline \multirow{4}{*}{ Comb. } & $\log _{10}(\bar{f})$ & $0.00 \pm 0.14$ & $0.43 \pm 0.09$ & $0.57 \pm 0.07$ \\
& $\sigma_{\log _{10} f}$ & $0.46 \pm 0.12$ & $0.22 \pm 0.11$ & $0.14 \pm 0.10$ \\
& $\log _{10}(f)_{\text {pred }}$ & $0.00 \pm 0.50$ & $0.43 \pm 0.26$ & $0.57 \pm 0.19$ \\
\hline
\end{tabular}

Note. Summary of the scale factors calculated for the LAMP 2011 sample (L11) and combined LAMP $2011+$ P14 + G17 samples (Comb.). The mean scale factor is $\log _{10}(\bar{f})$, the dispersion is $\sigma_{\log _{10} f}$, and the mean and standard deviation of the posterior predictive distribution is $\log _{10}(f)_{\text {pred }}$.

The posterior predictive distribution gives the distribution from which new $f$ measurements are drawn and so is the appropriate distribution to use when converting new virial product measurements to black hole masses. The standard deviation of the posterior predictive distribution for $\log _{10}\left(f_{\mathrm{rms}, \sigma}\right)$ is 0.19 , which is half the intrinsic scatter introduced by the $M_{\mathrm{BH}}-\sigma_{*}$ relation (Woo et al. 2010). It is possible that the small uncertainty is due to the narrow range in parameter space spanned by the sample used in this analysis, so future measurements will be necessary to solidify this result. Further, we find a similar value for $\log _{10}\left(f_{\text {mean }, \sigma}\right)$ as we do for $\log _{10}\left(f_{\text {rms, } \sigma}\right)$, with an intrinsic scatter that is only 0.26 . This suggests that the line dispersion can be measured in the mean spectrum when the rms spectrum is not readily available and still gives consistent black hole mass measurements. Since PG 1310-108 and Mrk 141 were not included in the calculation of $\log _{10}\left(f_{\text {rms }, \sigma}\right)_{\text {pred }}$, we re-do this analysis, excluding those two objects from the calculations of $\log _{10}\left(f_{\text {mean,FWHM }}\right)_{\text {pred }}$ and $\log _{10}\left(f_{\text {mean }, \sigma}\right)_{\text {pred }}$, and find that the scatters remain the same. This result is particularly useful in cases like PG 1310-108 and Mrk 141 where the rms spectrum does not have a sufficiently high signal-to-noise ratio to measure a line width.

\subsubsection{Correlations with Other AGN Properties}

The analysis described in this paper avoids the uncertainty introduced when using an average value for $f$ by modeling the BLR directly. Unfortunately, such an analysis requires highquality data sets from long and intensive observing campaigns, limiting its application to small samples of AGNs. However, using the scale factors found from the direct modeling approach, we can look for correlations between the scale factor and other AGN and BLR properties. If any exist, this would enable more precise measurements of the scale factor for individual AGNs based on observables.

In Figure 14, we examine correlations between the scale factor $f$ and various model parameters and other observables. Our calculation of the scale factor depends on the black hole mass inferred by the model, so the uncertainties in the scale factor are intrinsically tied to the uncertainties in other model parameters and values calculated using those parameters. Further, owing to degeneracies in the model, the uncertainties in other parameters are also correlated with the uncertainties in $f$. Correlated measurement uncertainties, if not taken into account, will increase (decrease) the measured correlation between two parameters if the sign of the measurement uncertainty correlation is the same as (opposite of) the intrinsic correlation between the two variables. We use the IDL routine linmix_err (Kelly 2007) to perform a Bayesian linear regression that accounts for correlated measurement uncertainties. We should note that linmix_err assumes Gaussian uncertainties, so the full 2D posterior PDFs for each data point are not used. The resulting fits are given in Table 5.

To quantify the strength of correlation, we compare the median fit slope to the $1 \sigma$ uncertainty in the slope. We define the following levels of confidence in the existence of a correlation: $0-2 \sigma$, no evidence; $2 \sigma-3 \sigma$, marginal evidence; $3 \sigma-5 \sigma$, evidence; $>5 \sigma$, conclusive evidence.

Figure 14 suggests a possible correlation between $\log _{10}$ $\left(f_{\text {mean,FWHM }}\right)$ and $\log _{10}\left(M_{\mathrm{BH}} / M_{\odot}\right)$. The linear regression analysis finds the slope of the correlation to be $\beta=0.59_{-0.30}^{+0.30}$, putting it at the boundary between our definitions of "no evidence" and "marginal evidence." The presence of this correlation would suggest that the mass of the black hole may influence the shape or dynamics of the BLR, but data spanning a larger range of black hole masses will help confirm or reject the presence of this correlation. There is no evidence of this correlation for $\log _{10}\left(f_{\text {mean }, \sigma}\right)$ or $\log _{10}\left(f_{\text {rms }, \sigma}\right)$.

There is also an apparent correlation between $\log _{10}\left(f_{\text {mean, } \sigma}\right)$ and $\log _{10}\left(f_{\text {rms, } \sigma}\right)$ and the Eddington fraction in Figure 14. One might expect this correlation to exist if the accretion rate has a strong influence on the BLR dynamics. However, since both values are calculated using $M_{\mathrm{BH}}$, the measurement uncertainties are highly correlated. When this is taken into account, there is no evidence of a correlation between the scale factor and Eddington fraction. We also find no evidence of a correlation between the scale factor and the AGN continuum luminosity at $5100 \AA$, $L_{5100}$, the uncertainties of which are independent of the uncertainties in $f$.

We do find marginal evidence for a negative correlation between $\log _{10}\left(f_{\text {mean }, \sigma}\right)$ and $\theta_{i}$, which was also found by P14 and G17. This result is unsurprising for models similar to ours and is predicted by Goad et al. (2012). For a given disk-like BLR, characteristic of the geometries that we measure, increasing the inclination angle has the effect of increasing the line-of-sight velocity and equivalently the measured line width $v$. This increases the virial product, requiring a smaller scale factor to recover the same black hole mass. While the negative correlation appears to also be strong for $\log _{10}\left(f_{\text {rms }, \sigma}\right)$ and $\log _{10}\left(f_{\text {mean,FWHM }}\right)$ in Figure 14, our full analysis finds no correlation, with $\beta=-0.030_{-0.019}^{+0.019}$ and $-0.033_{-0.021}^{+0.023}$, respectively.

An additional result of this analysis is a measurement of the intrinsic scatter in the relations between the scale factor and other parameters. We find that for every parameter, the median intrinsic scatter in $f_{\text {mean.FWHM }}$ is much higher than that for $f_{\text {mean }, \sigma}$ and $f_{\mathrm{rms}, \sigma}$, albeit with large error bars. This suggests that the line dispersion provides virial product measurements that are more tightly related to the true black hole mass. This result is also supported by the results of Section 5.2.1, in which the dispersion in the posterior predictive distribution is roughly half the size in the line-dispersion measurements as in the FWHM measurements. Thus, we suggest that line dispersion is a more meaningful measure of the line width and should be used when making $M_{\mathrm{BH}}$ measurements.

\section{Summary}

We have analyzed the data of seven AGNs from the Lick AGN Monitoring Project 2011 to constrain the structure and 


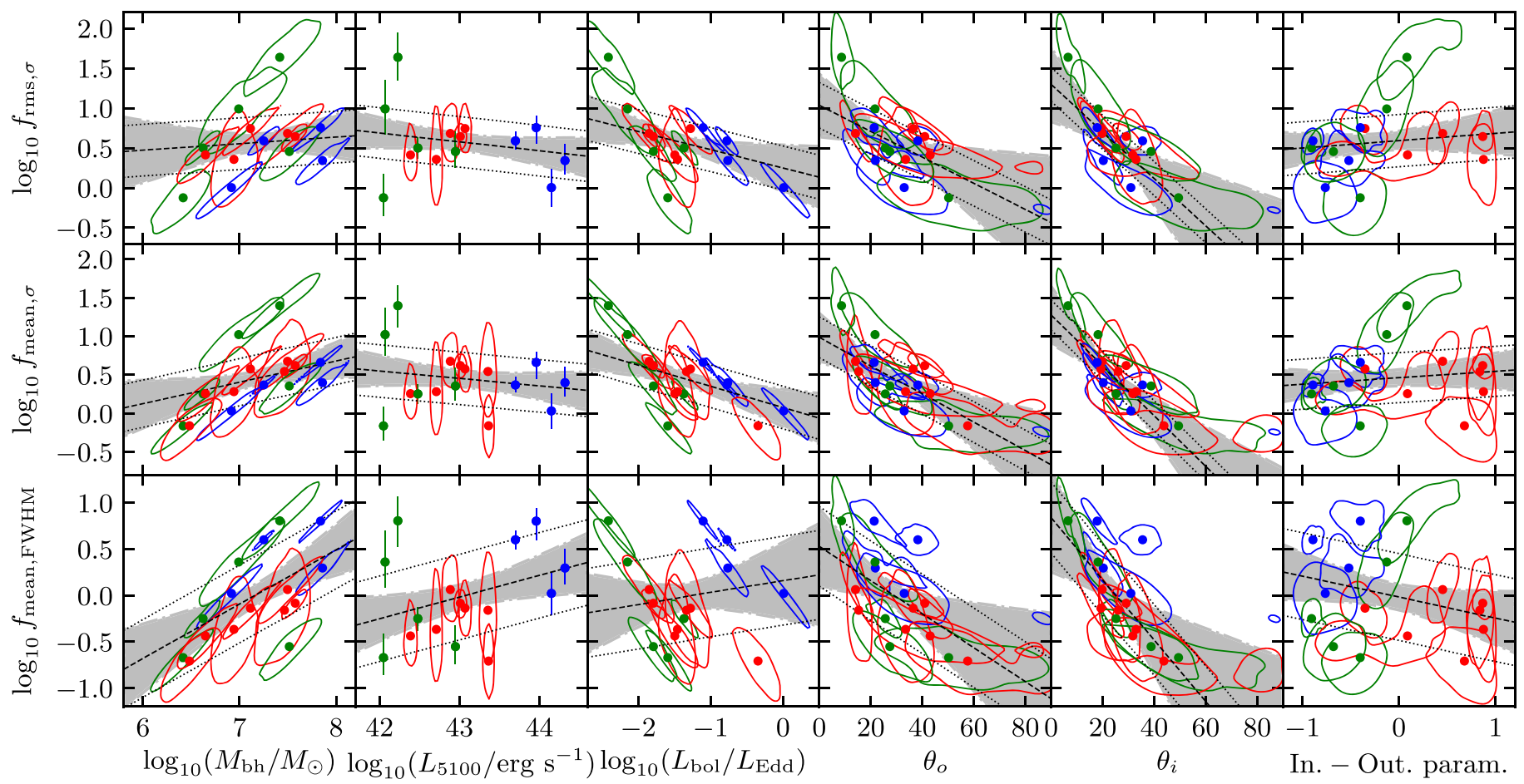

Figure 14. Correlations between the scale factor $f$ and select AGNs and model parameters. The colored dots and contours show the median and 68\% confidence regions of the 2D posterior PDFs for each AGN. When the abscissa uncertainty is unavailable, the 1D 68\% confidence interval is shown. The dashed black lines and gray shaded regions give the median and 68\% confidence intervals of the linear regression. Dotted lines are offset above and below the dashed line by the median value of the intrinsic scatter. Red points are for the AGNs in this paper, green points are from P14, and blue points are from G17.

Table 5

Linear Regression Results

\begin{tabular}{|c|c|c|c|c|c|c|c|}
\hline$f$-type & & $\log _{10}\left(M_{\mathrm{bh}} / M_{\odot}\right)$ & $\log _{10}\left(L_{5100} / \mathrm{erg} \mathrm{s}^{-1}\right)$ & $\log _{10}\left(L_{\mathrm{bol}} / L_{\mathrm{Edd}}\right)$ & $\theta_{o}$ (deg.) & $\theta_{i}$ (deg.) & In.-Out. param. \\
\hline \multirow[t]{4}{*}{ Mean, FWHM } & $\alpha$ & $-4.20_{-2.14}^{+2.18}$ & $-10.1_{-8.1}^{+8.3}$ & $0.16_{-0.41}^{+0.41}$ & $0.53_{-0.44}^{+0.41}$ & $0.84_{-0.59}^{+0.51}$ & $-0.02_{-0.14}^{+0.14}$ \\
\hline & $\beta$ & $0.59_{-0.30}^{+0.30}$ & $0.23_{-0.19}^{+0.19}$ & $0.13_{-0.29}^{+0.30}$ & $-0.018_{-0.014}^{+0.015}$ & $-0.033_{-0.021}^{+0.023}$ & $-0.23_{-0.24}^{+0.24}$ \\
\hline & $\sigma_{\text {int }}$ & $0.42_{-0.25}^{+0.34}$ & $0.46_{-0.30}^{+0.40}$ & $0.48_{-0.32}^{+0.41}$ & $0.43_{-0.26}^{+0.35}$ & $0.38_{-0.24}^{+0.32}$ & $0.47_{-0.30}^{+0.40}$ \\
\hline & $\alpha$ & $-1.53_{-1.89}^{+1.75}$ & $4.61_{-6.32}^{+6.71}$ & $0.08_{-0.25}^{+0.24}$ & $0.99_{-0.33}^{+0.31}$ & $1.28_{-0.38}^{+0.37}$ & $0.46_{-0.11}^{+0.10}$ \\
\hline \multirow[t]{3}{*}{ Mean, $\sigma$} & $\beta$ & $0.28_{-0.24}^{+0.26}$ & $-0.10_{-0.16}^{+0.15}$ & $-0.28_{-0.18}^{+0.17}$ & $-0.018_{-0.010}^{+0.011}$ & $-0.033_{-0.016}^{+0.015}$ & $0.08_{-0.18}^{+0.18}$ \\
\hline & $\sigma_{\text {int }}$ & $0.31_{-0.21}^{+0.28}$ & $0.34_{-0.24}^{+0.33}$ & $0.28_{-0.19}^{+0.26}$ & $0.26_{-0.18}^{+0.25}$ & $0.20_{-0.14}^{+0.20}$ & $0.32_{-0.24}^{+0.31}$ \\
\hline & $\alpha$ & $-0.01_{-2.34}^{+2.13}$ & $5.37_{-6.05}^{+6.53}$ & $0.25_{-0.30}^{+0.29}$ & $1.04_{-0.40}^{+0.39}$ & $1.30_{-0.47}^{+0.46}$ & $0.59_{-0.12}^{+0.12}$ \\
\hline \multirow[t]{2}{*}{$\mathrm{rms}, \sigma$} & $\beta$ & $0.08_{-0.29}^{+0.32}$ & $-0.11_{-0.15}^{+0.14}$ & $-0.23_{-0.20}^{+0.20}$ & $-0.016_{-0.013}^{+0.013}$ & $-0.030_{-0.019}^{+0.019}$ & $0.09_{-0.19}^{+0.21}$ \\
\hline & $\sigma_{\text {int }}$ & $0.32_{-0.25}^{+0.34}$ & $0.32_{-0.25}^{+0.35}$ & $0.28_{-0.21}^{+0.29}$ & $0.28_{-0.21}^{+0.29}$ & $0.21_{-0.16}^{+0.23}$ & $0.33_{-0.25}^{+0.35}$ \\
\hline
\end{tabular}

Note. Median values and $68 \%$ confidence intervals for the main BLR geometry and dynamics model parameters. Upper and lower $68 \%$ confidence limits are given when the posterior PDF is one-sided. Note that $r_{\text {out }}$ is a fixed parameter, so we do not include uncertainties.

dynamics of the $\mathrm{H} \beta$-emitting BLR. Our results can be summarized as follows.

1. The $\mathrm{H} \beta$-emitting BLR is best described by a thick disk that is closer to face-on than edge-on, with a radial distribution that is between Gaussian and exponential, in agreement with the results for the BLRs in P14 and G17. The $\mathrm{H} \beta$ emission comes preferentially from the far side of the BLR, which is consistent with photoionization modeling predictions.

2. Dynamically, the BLR gas can be on elliptical orbits, inflowing or outflowing motions, or a combination of elliptical orbits and either inflowing or outflowing motions. The preference for outflowing gas in many of the AGNs is a result that has not been seen in the BLR of other AGNs that have been modeled in this manner.

3. We measure black hole masses of $\log _{10}\left(M_{\mathrm{BH}} / M_{\odot}\right)=$ $6.48_{-0.18}^{+0.21}$ for PG 1310-108, 7.50 $0_{-0.18}^{+0.25}$ for Mrk 50, $7.46_{-0.21}^{+0.15}$ for Mrk 141, 7.58 $8_{-0.08}^{+0.08}$ for Mrk 279, 7.11 $1_{-0.17}^{+0.20}$ for Mrk 1511, 6.65 -0.15 for NGC 4593, and $6.94_{-0.14}^{+0.14}$ for $\mathrm{Zw}$ 229-015. All values are fully consistent with previous measurements, except for that of PG 1310-108. However, this discrepancy may be due to additional uncertainties in the single-epoch method of black hole mass estimates.

4. We measure a mean scale factor for the LAMP 2011 sample of $\log _{10}\left(f_{\text {rms }, \sigma}\right)=0.60 \pm 0.16$. Combined with the results from P14 and G17, we find $\log _{10}\left(f_{\text {rms }, \sigma}\right)=0.57 \pm 0.07$. 
The posterior predictive distribution for $f$ shows little scatter and can be used to measure black hole masses with other reverberation mapping data: $\log _{10}\left(f_{\text {rms }, \sigma}\right)=0.57 \pm 0.19$. Further, the agreement between these values and the scale factors found by aligning AGNs with the $M_{\mathrm{BH}}-\sigma_{*}$ relation for quiescent galaxies indicates that the $M_{\mathrm{BH}}-\sigma_{*}$ relation for AGN is consistent with that for quiescent galaxies.

5. The scale factors we recover when using the line dispersion measured in the mean spectrum are consistent with those found when the rms spectrum is used. The scatter in the posterior predictive distribution is of similar magnitude, showing that the mean spectrum is a suitable alternative when the rms spectrum is either unavailable or does not have a sufficient signal-to-noise ratio to measure a line width: $\log _{10}\left(f_{\text {mean }, \sigma}\right)_{\text {pred }}=0.43 \pm 0.26$. When the FWHM is used instead of the line dispersion, we find the largest scatter, with $\log _{10}\left(f_{\text {mean,FWHM }}\right)_{\text {pred }}=0.00 \pm 0.50$.

6. When the line width is measured as the FWHM in the mean spectrum, we find marginal evidence for a correlation between the scale factor $\log _{10}\left(f_{\text {mean,FWHM }}\right)$ and $\log _{10}\left(M_{\mathrm{BH}} / M_{\odot}\right)$. There is no significant correlation present when the line dispersion is used instead. There is also marginal evidence of a correlation between the scale factor and the inclination angle when the line dispersion is used, measured in the mean spectrum. We find no significant correlation between the scale factor and the AGN continuum luminosity or Eddington ratio.

The modeling of these objects from the LAMP 2011 sample has nearly doubled the number of AGNs with dynamical modeling of the BLR. The increased sample has allowed us to measure predictive values of $f$ with scatter that is now smaller than that of the $M_{\mathrm{BH}}-\sigma_{*}$ relation. These results can be used with traditional reverberation mapping techniques to obtain more precise black hole mass measurements. However, the significance of correlations between the scale factor and other AGN properties remains uncertain. Further analysis of data from other reverberation mapping campaigns covering a more diverse range of AGN properties will help uncover these correlations, which can then be used to make accurate $M_{\mathrm{BH}}$ measurements on an individual AGN basis.

We thank the Lick Observatory staff for their exceptional support during our observing campaign. The Kast spectrograph was made possible through a generous gift from William and Marina Kast. KAIT (at Lick) and its ongoing operation were made possible by donations from Sun Microsystems, Inc., the Hewlett-Packard Company, AutoScope Corporation, Lick Observatory, the National Science Foundation (NSF), the University of California, the Sylvia \& Jim Katzman Foundation, and the TABASGO Foundation. Research at Lick Observatory is partially supported by a generous gift from Google.

The Lick AGN Monitoring Project 2011 is supported by National Science Foundation (NSF) grants AST-1107812, 1107865, 1108665, and 1108835. (Note that findings and conclusions do not necessarily represent views of the NSF.) Research by P.R.W. and T.T. is supported by NSF grant AST1412315. B.J.B. and T.T. acknowledge support from the Packard Foundation through a Packard Fellowship to T.T. Research by A.J.B. is supported by NSF grant AST-1412693. The West Mountain Observatory $0.9 \mathrm{~m}$ telescope was supported by NSF grant AST0618209 during this campaign.
M.D.J. would like to thank the Department of Physics and Astronomy at Brigham Young University for continued support of the research efforts at the West Mountain Observatory. V.N.B. gratefully acknowledges assistance from an NSF Research at Undergraduate Institutions (RUI). grant AST-1312296. A.V.F. has been supported by the Christopher R. Redlich Fund, the TABASGO Foundation, NSF grant AST1211916, and the Miller Institute for Basic Research in Science (UC Berkeley). His work was conducted in part at the Aspen Center for Physics, which is supported by NSF grant PHY1607611; he thanks the Center for its hospitality during the supermassive black holes workshop in June and July 2018. S.F.H. is supported by European Research Council Starting grant ERC-StG-677117 DUST-IN-THE-WIND. J.-H.W. acknowledges the support by the National Research Foundation of Korea (NRF) grant funded by the Korea government (No. 2017R1A5A1070354).

\section{ORCID iDs}

Peter R. Williams (1) https://orcid.org/0000-0002-4645-6578 Anna Pancoast (D) https://orcid.org/0000-0003-1065-5046 Tommaso Treu (iD https://orcid.org/0000-0002-8460-0390 Aaron J. Barth (i) https://orcid.org/0000-0002-3026-0562 Vardha N. Bennert (iD https://orcid.org/0000-0003-2064-0518 S. Bradley Cenko (iD https://orcid.org/0000-0003-1673-970X Michael C. Cooper (iD https://orcid.org/0000-0003-1371-6019 Alexei V. Filippenko (iD https://orcid.org/0000-0003-3460-0103 Sebastian F. Hoenig (10) https://orcid.org/0000-0002-6353-1111 Clifton David Laney (iD https://orcid.org/0000-0003-2967-110X Matthew A. Malkan (iD https://orcid.org/0000-0001-6919-1237 Jeffrey M. Silverman (iD https://orcid.org/0000-0003-3325-3365 Erik Tollerud (iD https://orcid.org/0000-0002-9599-310X Jonelle L. Walsh (iD https://orcid.org/0000-0002-1881-5908 Jong-Hak Woo (iD https://orcid.org/0000-0002-8055-5465

\section{References}

Barth, A. J., Bennert, V. N., Canalizo, G., et al. 2015, ApJS, 217, 26 Barth, A. J., Nguyen, M. L., Malkan, M. A., et al. 2011a, ApJ, 732, 121 Barth, A. J., Pancoast, A., Thorman, S. J., et al. 2011b, ApJL, 743, L4 Batiste, M., Bentz, M. C., Raimundo, S. I., Vestergaard, M., \& Onken, C. A. 2017, ApJL, 838, L10

Bentz, M. C., Horne, K., Barth, A. J., et al. 2010, ApJL, 720, L46

Bentz, M. C., Peterson, B. M., Netzer, H., Pogge, R. W., \& Vestergaard, M. 2009a, ApJ, 697, 160

Bentz, M. C., Walsh, J. L., Barth, A. J., et al. 2009b, ApJ, 705, 199

Blandford, R. D., \& McKee, C. F. 1982, ApJ, 255, 419

Boroson, T. A., \& Green, R. F. 1992, ApJS, 80, 109

Brewer, B. J., Pártay, L. B., \& Csányi, G. 2011a, Stat. Comp., 21, 649

Brewer, B. J., Treu, T., Pancoast, A., et al. 2011b, ApJL, 733, L33

Brown, T. M., Baliber, N., Bianco, F. B., et al. 2013, PASP, 125, 1031

Busch, G., Zuther, J., Valencia-S., M., et al. 2014, A\&A, 561, A140

Castelló-Mor, N., Kaspi, S., Netzer, H., et al. 2017, MNRAS, 467, 1209

Cenko, S. B., Fox, D. B., Moon, D.-S., et al. 2006, PASP, 118, 1396

Collin, S., Kawaguchi, T., Peterson, B. M., \& Vestergaard, M. 2006, A\&A, 456, 75

Denney, K. D., Peterson, B. M., Dietrich, M., Vestergaard, M., \& Bentz, M. C. 2009a, ApJ, 692, 246

Denney, K. D., Peterson, B. M., Pogge, R. W., et al. 2009b, ApJL, 704, L80 Denney, K. D., Peterson, B. M., Pogge, R. W., et al. 2010, ApJ, 721, 715

Du, P., Lu, K.-X., Hu, C., et al. 2016, ApJ, 820, 27

Ferland, G. J., Peterson, B. M., Horne, K., Welsh, W. F., \& Nahar, S. N. 1992, ApJ, 387, 95

Ferrarese, L., \& Ford, H. 2005, SSRv, 116, 523

Ferrarese, L., \& Merritt, D. 2000, ApJL, 539, L9

Filippenko, A. V., Li, W. D., Treffers, R. R., \& Modjaz, M. 2001, in ASP Conf. Ser. 246, IAU Colloq. 183: Small Telescope Astronomy on Global 
Scales, ed. B. Paczynski, W.-P. Chen, \& C. Lemme (San Francisco, CA: ASP), 121

Gebhardt, K., Bender, R., Bower, G., et al. 2000, ApJL, 539, L13

Goad, M. R., Korista, K. T., \& Ruff, A. J. 2012, MNRAS, 426, 3086

Graham, A. W., Onken, C. A., Athanassoula, E., \& Combes, F. 2011, MNRAS, 412, 2211

Grier, C. J., Martini, P., Watson, L. C., et al. 2013a, ApJ, 773, 90

Grier, C. J., Pancoast, A., Barth, A. J., et al. 2017, ApJ, 849, 146

Grier, C. J., Peterson, B. M., Horne, K., et al. 2013b, ApJ, 764, 47

Horne, K. 1994, in ASP Conf. Ser. 69, Reverberation Mapping of the Broad-

Line Region in Active Galactic Nuclei, ed. P. M. Gondhalekar, K. Horne, \& B. M. Peterson (San Francisco, CA: ASP), 23

Horne, K., Welsh, W. F., \& Peterson, B. M. 1991, ApJL, 367, L5

Kelly, B. C. 2007, ApJ, 665, 1489

Kelly, B. C., Bechtold, J., \& Siemiginowska, A. 2009, ApJ, 698, 895

Kormendy, J., \& Richstone, D. 1995, ARA\&A, 33, 581

Kovačević, J., Popović, L. Č., \& Dimitrijević, M. S. 2010, ApJS, 189, 15

Kozłowski, S. 2016, ApJ, 826, 118

Kozłowski, S., Kochanek, C. S., Udalski, A., et al. 2010, ApJ, 708, 927

Li, Y.-R., Wang, J.-M., Ho, L. C., Du, P., \& Bai, J.-M. 2013, ApJ, 779, 110

Li, Z.-Y., Wu, X.-B., \& Wang, R. 2008, ApJ, 688, 826

MacLeod, C. L., Ivezić, Ž, Kochanek, C. S., et al. 2010, ApJ, 721, 1014

Magorrian, J., Tremaine, S., Richstone, D., et al. 1998, AJ, 115, 2285

Maoz, D., Netzer, H., Leibowitz, E., et al. 1990, ApJ, 351, 75

Marconi, A., Axon, D. J., Maiolino, R., et al. 2008, ApJ, 678, 693

Marconi, A., Axon, D. J., Maiolino, R., et al. 2009, ApJL, 698, L103
Markwardt, C. B. 2009, in ASP Conf. Ser. 411, Astronomical Data Analysis Software and Systems XVIII, ed. D. A. Bohlender, D. Durand, \& P. Dowler (San Francisco, CA: ASP), 251

Netzer, H. 2009, ApJ, 695, 793

Netzer, H., \& Marziani, P. 2010, ApJ, 724, 318

O’Brien, P. T., Goad, M. R., \& Gondhalekar, P. M. 1994, MNRAS, 268, 845

Onken, C. A., Ferrarese, L., Merritt, D., et al. 2004, ApJ, 615, 645

Pancoast, A., Brewer, B. J., \& Treu, T. 2011, ApJ, 730, 139

Pancoast, A., Brewer, B. J., Treu, T., et al. 2012, ApJ, 754, 49

Pancoast, A., Brewer, B. J., \& Treu, T. 2014a, MNRAS, 445, 3055

Pancoast, A., Brewer, B. J., Treu, T., et al. 2014b, MNRAS, 445, 3073

Park, D., Kelly, B. C., Woo, J.-H., \& Treu, T. 2012, ApJS, 203, 6

Pei, L., Fausnaugh, M. M., Barth, A. J., et al. 2017, ApJ, 837, 131

Peterson, B. M. 1993, PASP, 105, 247

Peterson, B. M., Ferrarese, L., Gilbert, K. M., et al. 2004, ApJ, 613, 682

Santos-Lleó, M., Clavel, J., Schulz, B., et al. 2001, A\&A, 369, 57

Schulze, A., \& Wisotzki, L. 2010, A\&A, 516, A87

Shen, Y., \& Kelly, B. C. 2010, ApJ, 713, 41

Skielboe, A., Pancoast, A., Treu, T., et al. 2015, MNRAS, 454, 144

van Groningen, E., \& Wanders, I. 1992, PASP, 104, 700

Véron-Cetty, M.-P., Joly, M., \& Véron, P. 2004, A\&A, 417, 515

Walsh, J. L., Minezaki, T., Bentz, M. C., et al. 2009, ApJS, 185, 156

Welsh, W. F., \& Horne, K. 1991, ApJ, 379, 586

Whittle, M. 1992, ApJS, 79, 49

Woo, J.-H., Schulze, A., Park, D., et al. 2013, ApJ, 772, 49

Woo, J.-H., Treu, T., Barth, A. J., et al. 2010, ApJ, 716, 269

Zu, Y., Kochanek, C. S., Kozłowski, S., \& Udalski, A. 2013, ApJ, 765, 106

Zu, Y., Kochanek, C. S., \& Peterson, B. M. 2011, ApJ, 735, 80 